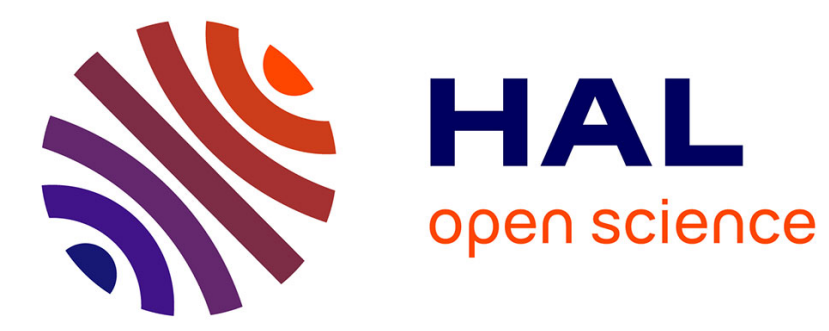

\title{
Definability and canonicity for Boolean logic with a binary relation
}

Philippe Balbiani, Tinko Tinchev

\section{To cite this version:}

Philippe Balbiani, Tinko Tinchev. Definability and canonicity for Boolean logic with a binary relation. Fundamenta Informaticae, 2014, vol. 129 ( ${ }^{\circ}$ 4), pp. 301-327. 10.3233/FI-2014-973 . hal-01120594

\author{
HAL Id: hal-01120594 \\ https://hal.science/hal-01120594
}

Submitted on 26 Feb 2015

HAL is a multi-disciplinary open access archive for the deposit and dissemination of scientific research documents, whether they are published or not. The documents may come from teaching and research institutions in France or abroad, or from public or private research centers.
L'archive ouverte pluridisciplinaire HAL, est destinée au dépôt et à la diffusion de documents scientifiques de niveau recherche, publiés ou non, émanant des établissements d'enseignement et de recherche français ou étrangers, des laboratoires publics ou privés. 


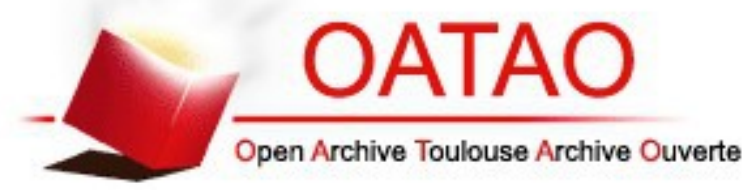

\section{Open Archive TOULOUSE Archive Ouverte (OATAO)}

OATAO is an open access repository that collects the work of Toulouse researchers and makes it freely available over the web where possible.

This is an author-deposited version published in : http://oatao.univ-toulouse.fr/ Eprints ID : 12876

To link to this article : DOI :10.3233/FI-2014-973

URL : http://dx.doi.org/10.3233/FI-2014-973

To cite this version : Balbiani, Philippe and Tinchev, Tinko Definability and canonicity for Boolean logic with a binary relation. (2014) Fundamenta Informaticae, vol. 129 (n 4). pp. 301-327. ISSN 0169-2968

Any correspondance concerning this service should be sent to the repository administrator: staff-oatao@,listes-diff.inp-toulouse.fr 


\title{
Definability and Canonicity for Boolean Logic with a Binary Relation
}

\author{
Philippe Balbiani* \\ Institut de recherche en informatique de Toulouse, CNRS - Université de Toulouse \\ 118 route de Narbonne, 31062 Toulouse Cedex 9, France \\ Philippe.Balbiani@irit.fr
}

Tinko Tinchev

Department of Mathematical Logic and Applications, Sofia University

Blvd James Bouchier 5, 1126 Sofia, Bulgaria

tinko@fmi.uni-sofia.bg

\begin{abstract}
This paper studies the concepts of definability and canonicity in Boolean logic with a binary relation. Firstly, it provides formulas defining first-order or second-order conditions on frames. Secondly, it proves that all formulas corresponding to compatible first-order conditions on frames are canonical.
\end{abstract}

Keywords: Boolean algebra, modal logic, definability, canonicity.

\section{Introduction}

One of the central issues in region-based theories of space is the concept of connection between regions $[10,11,17,18]$. The theory of connection can be succinctly described as the study of regions instead of points as the basic entities of geometry, with a particular emphasis on the study of the relation " $a$ is in contact with $b$ " for regions $a$ and $b$ in some space. For example, de Laguna [22] considers the ternary relation " $a$ connects $b$ with $c$ " and Whitehead [31] considers the binary relation " $a$ is connected with $b$ ". In this setting, points can be defined as collections of regions. The idea to define points as collections of regions in de Laguna's framework and in Whitehead's framework is very similar to

*Address for correspondence: Institut de recherche en informatique de Toulouse, CNRS — Université de Toulouse, 118 route de Narbonne, 31062 Toulouse Cedex 9, France 
the idea to define elements as ultrafilters in Stone's representation theory of Boolean algebras. That is the reason why the concept of connection can be abstracted within the context of Boolean contact algebras where one considers a Boolean algebra $B$, the space of regions, and a binary relation $C$ on $B$, the contact relation between regions. Several variants of Boolean contact algebras have been studied later $[6,15,21,26,29]$ and several representation theorems of these variants in proximity spaces and topological spaces have been recently obtained $[12,13,14,16,28]$.

Boolean logic with a binary relation (BLBR) can be considered as a quantifier-free logic for reasoning about connection between regions $[3,4,5]$. Its language is a Boolean language with the Boolean constructs $f, t,-, \cup$ and $\cap$ to which the binary relation symbol $\delta$ has been added. Within a Boolean contact algebra $(B, C)$, the Boolean terms of the language defined over $f, t,-, \cup$ and $\cap$ are interpreted by elements of the Boolean algebra $B$ whereas the binary relation symbol $\delta$ added to the language is interpreted by the binary relation $C$ on $B$. In spite of its simplicity, such a language turns out to be a useful tool for describing relational structures [2] too. The truth of the matter is that the semantics of BLBR can be presented in three different ways [27]: an algebraic semantics based on algebras of regions, a topological semantics based on contact algebras of some classes of topological spaces and a Kripke-type semantics based on Kripke structures regarded as adjancency spaces, the Kripke-type semantics having the advantage of being close to the semantics for basic modal language and allowing the re-use of well-known tools and techniques in modal logic (bisimulation, canonical model, filtration, etc).

This paper considers the concepts of definability and canonicity. It presents results that explain the differences between our BLBR and a propositional modal logic. Concerning definability, we show that the class of all connected frames is modally definable in our language whereas the class of all Church-Rosser frames is not modally definable in our language. Concerning canonicity, a consistent extension $L$ of the minimal BLBR is said to be canonical iff every maximal $L$-consistent set of formulas in our language defines a canonical frame that validates $L$. The most important differences between our BLBR and a propositional modal logic being probably that our BLBR gives rise to uncountably many canonical models, we show that some consistent extension $L$ of our BLBR is not canonical.

The section-by-section breakdown of this paper is as follows. Section 2 introduces the syntax and the Kripke-type semantics of our BLBR. In Section 3, we redefine in our setting the concepts of bounded morphisms and bisimulations. Section 4 considers the concept of definability. A variant of the technique of the filtration is presented in Section 5. Section 6 describes a variant of the technique of the canonical model. In Section 7, the concept of canonicity is studied. Section 8 presents the concept of compatible formula. In Section 9, several open problems are suggested. We assume the reader's familiarity with well-known tools and techniques in modal logic (bisimulation, canonical model, filtration, etc). For more on these see [7,9]. In all our figures, true to tradition, black circles represent irreflexive possible worlds whereas white circles represent reflexive possible worlds.

\section{Syntax and Kripke-type semantics}

We now recall the syntax and the Kripke-type semantics presented in [5].

\subsection{Syntax}

The language is defined using a countable set $B V$ of Boolean variables (with typical members denoted by $p, q, r$, etc). 


\section{Definition 2.1. (Terms)}

We inductively define the set $t(B V)$ of terms (with typical members denoted by $A, B, C$, etc) as follows:

- $A::=p|f| t|-A|(A \cup B) \mid(A \cap B)$.

For all terms $A$, let $A^{0}=-A$ and $A^{1}=A$.

\section{Definition 2.2. (Formulas)}

We inductively define the set $f(B V)$ of formulas (with typical members denoted by $\phi, \psi$, $\chi$, etc) as follows:

- $\phi::=A \equiv B|A \delta B| \perp|\top| \neg \phi|(\phi \vee \psi)|(\phi \wedge \psi)$.

The other Boolean constructs are defined as usual. We obtain the formulas $A \not \equiv B$ and $A \bar{\delta} B$ as abbreviations: $A \not \equiv B$ for $\neg A \equiv B$ and $A \bar{\delta} B$ for $\neg A \delta B$. The notion of subterm and the notion of subformula are standard. We adopt the standard rules for omission of the parentheses.

\section{Definition 2.3. (Free variables)}

If $A$ is a term then $F V(A)$ will denote the set of all Boolean variables occurring in $A$ whereas if $\phi$ is a formula then $F V(\phi)$ will denote the set of all Boolean variables occurring in $\phi$. For all $B V^{\prime} \subseteq B V$, $t\left(B V^{\prime}\right)$ will denote the set of all $A \in t(B V)$ such that $F V(A) \subseteq B V^{\prime}$ whereas $f\left(B V^{\prime}\right)$ will denote the set of all $\phi \in f(B V)$ such that $F V(\phi) \subseteq B V^{\prime}$.

\section{Definition 2.4. (Substitution instances)}

A substitution is a function $\sigma$ assigning to each Boolean variable $p$ a term $\sigma(p)$. As usual, $\sigma$ induces a function $(\cdot)^{\sigma}$ assigning to each term $A$ a term $(A)^{\sigma}$ and assigning to each formula $\phi$ a formula $(\phi)^{\sigma}$ obtained from $A$ and $\phi$ by uniformly replacing occurrences of Boolean variables by the $\sigma$-corresponding terms. A term $B$ is a substitution instance of a term $A$ iff there exists a substitution $\sigma$ such that $(A)^{\sigma}=B$ whereas a formula $\psi$ is a substitution instance of a formula $\phi$ iff there exists a substitution $\sigma$ such that $(\phi)^{\sigma}=\psi$

\subsection{Kripke-type semantics}

\section{Definition 2.5. (Frames)}

A frame is an ordered pair $\mathcal{F}=(W, R)$ where $W$ is a non-empty set of possible worlds and $R$ is a binary relation on $W$. For all $x \in W$, let $R(x)$ be the set of all $y \in W$ such that $x R y, R^{+}(x)$ be the set of all $y \in W$ such that $x R^{+} y$ and $R^{\star}(x)$ be the set of all $y \in W$ such that $x R^{\star} y, R^{+}$being the transitive closure of $R$ and $R^{\star}$ being the reflexive-transitive closure of $R$.

\section{Definition 2.6. (Valuations)}

A valuation based on a frame $\mathcal{F}=(W, R)$ is a function $V$ assigning to each Boolean variable $p$ a subset $V(p)$ of $W$. As usual, $V$ induces a homomorphism $(\cdot)^{V}$ from the algebra of terms into the Boolean algebra of $W$ 's subsets assigning to each term $A$ a subset $(A)^{V}$ of $W$ as follows:

- $(p)^{V}=V(p)$,

- $(f)^{V}=\emptyset$,

- $(t)^{V}=W$, 
- $(-A)^{V}=W \backslash(A)^{V}$,

- $(A \cup B)^{V}=(A)^{V} \cup(B)^{V}$ and

- $(A \cap B)^{V}=(A)^{V} \cap(B)^{V}$.

\section{Definition 2.7. (Models)}

A model is an ordered triple $\mathcal{M}=(W, R, V)$ where $\mathcal{F}=(W, R)$ is a frame and $V$ is a valuation based on $\mathcal{F}$.

\section{Definition 2.8. (Satisfiability)}

The satisfiability of a formula $\phi$ in a model $\mathcal{M}=(W, R, V)$, in symbols $\mathcal{M} \models \phi$, is defined as follows:

- $\mathcal{M} \models A \equiv B$ iff $(A)^{V}=(B)^{V}$,

- $\mathcal{M} \models A \delta B$ iff there exists $x, y \in W$ such that $x R y, x \in(A)^{V}$ and $y \in(B)^{V}$,

- $\mathcal{M} \not \models \perp$,

- $\mathcal{M} \models \top$,

- $\mathcal{M} \models \neg \phi$ iff $\mathcal{M} \not \models \phi$,

- $\mathcal{M} \models \phi \vee \psi$ iff $\mathcal{M} \models \phi$ or $\mathcal{M} \models \psi$ and

- $\mathcal{M} \models \phi \wedge \psi$ iff $\mathcal{M} \models \phi$ and $\mathcal{M} \models \psi$.

As a result, $\mathcal{M} \models A \not \equiv B$ iff $(A)^{V} \neq(B)^{V}$ and $\mathcal{M} \models A \bar{\delta} B$ iff for all $x, y \in W$, if $x R y$ then $x \notin(A)^{V}$ or $y \notin(B)^{V}$.

Example 2.9. Let $\mathcal{M}=(W, R, V)$ and $\mathcal{M}^{\prime}=\left(W^{\prime}, R^{\prime}, V^{\prime}\right)$ be the models defined as follows: $W=$ $\{0\} ; R=\emptyset ; V(p)=\emptyset ; W^{\prime}=\{0\} ; R^{\prime}=\emptyset ; V^{\prime}(p)=\{0\}$. The reader may easily verify that $\equiv$ cannot be eliminated from the language, seeing that $\mathcal{M} \models p \equiv f, \mathcal{M}^{\prime} \not \models p \equiv f$ and for all $\equiv$-free formulas $\phi$, $\mathcal{M} \models \phi$ iff $\mathcal{M}^{\prime} \models \phi$.

Example 2.10. Let $\mathcal{M}=(W, R, V)$ and $\mathcal{M}^{\prime}=\left(W^{\prime}, R^{\prime}, V^{\prime}\right)$ be the models defined as follows: $W=$ $\{0\} ; R=\emptyset ; V(p)=\emptyset ; W^{\prime}=\{0\} ; R^{\prime}=\{(0,0)\} ; V^{\prime}(p)=\emptyset$. The reader may easily verify that $\delta$ cannot be eliminated from the language, seeing that $\mathcal{M} \models t \bar{\delta} t, \mathcal{M}^{\prime} \not t \bar{\delta} t$ and for all $\delta$-free formulas $\phi$, $\mathcal{M} \models \phi$ iff $\mathcal{M}^{\prime} \models \phi$.

\section{Definition 2.11. (Validity and satisfiability)}

Let $\mathcal{F}$ be a frame. A formula $\phi$ is valid in $\mathcal{F}$, in symbols $\operatorname{val}(\mathcal{F}, \phi)$, iff for all models $\mathcal{M}$ based on $\mathcal{F}$, $\mathcal{M} \models \phi$. If there exists a model $\mathcal{M}$ based on $\mathcal{F}$ such that $\mathcal{M} \models \phi$ then we say that $\phi$ is satisfiable in $\mathcal{F}$, in symbols $\operatorname{sat}(\mathcal{F}, \phi)$. A set $\Gamma$ of formulas is valid in $\mathcal{F}$, in symbols $\operatorname{val}(\mathcal{F}, \Gamma)$, iff for all models $\mathcal{M}$ based on $\mathcal{F}, \mathcal{M} \models \phi$ for every formula $\phi$ such that $\phi \in \Gamma$. If there exists a model $\mathcal{M}$ based on $\mathcal{F}$ such that $\mathcal{M} \models \phi$ for every formula $\phi$ such that $\phi \in \Gamma$ then we say that $\Gamma$ is satisfiable in $\mathcal{F}$, in symbols $\operatorname{sat}(\mathcal{F}, \Gamma)$. Let $\mathcal{C}$ be a class of frames. A formula $\phi$ is valid in $\mathcal{C}$, in symbols $\operatorname{val}(\mathcal{C}, \phi)$, iff for all frames $\mathcal{F}$ in $\mathcal{C}, \operatorname{val}(\mathcal{F}, \phi) . \phi$ is said to be valid if $\phi$ is valid in the class of all frames. If there exists a frame $\mathcal{F}$ in $\mathcal{C}$ such that $\operatorname{sat}(\mathcal{F}, \phi)$ then we say that $\phi$ is satisfiable in $\mathcal{C}$, in symbols $\operatorname{sat}(\mathcal{C}, \phi) . \phi$ is said to be satisfiable if $\phi$ is satisfiable in the class of all frames. For all classes $\mathcal{C}$ of frames, let $\operatorname{val}(\mathcal{C})$ be the set of all formulas $\phi$ such that $\operatorname{val}(\mathcal{C}, \phi)$ and $\operatorname{sat}(\mathcal{C})$ be the set of all formulas $\phi$ such that $\operatorname{sat}(\mathcal{C}, \phi)$. For all formulas $\phi$, let $\mathcal{C}_{\phi}^{v a l}$ be the class of all frames $\mathcal{F}$ such that $\operatorname{val}(\mathcal{F}, \phi)$ and $\mathcal{C}_{\phi}^{\text {sat }}$ be the class of all frames $\mathcal{F}$ such that $\operatorname{sat}(\mathcal{F}, \phi)$. 


\section{Definition 2.12. (Modal equivalence)}

Let $\mathcal{M}=(W, R, V)$ and $\mathcal{M}^{\prime}=\left(W^{\prime}, R^{\prime}, V^{\prime}\right)$ be models. If $\mathcal{M}$ and $\mathcal{M}^{\prime}$ are such that for all formulas $\phi$, $\mathcal{M} \models \phi$ iff $\mathcal{M}^{\prime} \models \phi$ then we say that $\mathcal{M}$ and $\mathcal{M}^{\prime}$ are modally equivalent.

Example 2.13. Take the case of the models $\mathcal{M}_{\text {fin }}=\left(W_{\text {fin }}, R_{\text {fin }}, V_{\text {fin }}\right)$ and $\mathcal{M}_{\text {inf }}=\left(W_{\text {inf }}, R_{\text {inf }}\right.$, $\left.V_{\text {inf }}\right)$ defined as follows: $W_{\text {fin }}$ is the set of all finite subsets of $B V ; R_{f i n}$ is the universal relation on $W_{\text {fin }}$; for all Boolean variables $p$ and for all $x \in W_{\text {fin }}, x \in V_{\text {fin }}(p)$ iff $p \in x$; $W_{\text {inf }}$ is the set of all infinite subsets of $B V ; R_{\text {inf }}$ is the universal relation on $W_{\text {inf }}$; for all Boolean variables $p$ and for all $x \in W_{\text {inf }}, x \in V_{\text {inf }}(p)$ iff $p \in x$. As the reader is asked to show, $\mathcal{M}_{\text {fin }}$ and $\mathcal{M}_{\text {inf }}$ are modally equivalent.

\subsection{Standard translation into a first-order language}

By now, the reader should have noticed an important difference between the above Kripke-type semantics and the semantics for the basic modal language: in the above Kripke-type semantics, satisfaction is a binary relation between models and formulas whereas in the semantics for the basic modal language, satisfaction is a ternary relation between models, possible worlds and formulas. Such a difference relates to the way we have defined the satisfiability of the formulas $A \equiv B$ and $A \delta B$ in models. This way implies that in every model, the operators $[U]$ and $\langle U\rangle$ being interpreted by the universal binary relation on the set of all possible worlds and the operators $\square$ and $\diamond$ being interpreted by the binary relation $R$ on the set of all possible worlds, $A \equiv B$ corresponds to $[U](A \leftrightarrow B)$ and $A \delta B$ corresponds to $\langle U\rangle(A \wedge \diamond B)$. The following translation of our language into a first-order language illustrates this correspondence. Let $\mathcal{L}^{1}(B V)$ be the first-order language with equality which has the unary predicates $P_{0}, P_{1}, \ldots$ corresponding to the Boolean variables $p_{0}, p_{1}, \ldots$ in $B V$ and the binary predicate $R_{\delta}$ corresponding to the modal operator $\delta$ and $\mathcal{L}^{1}(\emptyset)$ be the first-order language with equality which has the binary predicate $R_{\delta}$ corresponding to the modal operator $\delta$. Positive first-order formulas in $\mathcal{L}^{1}(\emptyset)$ are inductively defined as follows:

- $\alpha::=u \equiv v\left|R_{\delta}(u, v)\right|(\alpha \vee \beta)|(\alpha \wedge \beta)| \forall u \alpha \mid \exists u \alpha$.

Quantifier-free first-order formulas in $\mathcal{L}^{1}(\emptyset)$ are inductively defined as follows:

- $\alpha::=u \equiv v\left|R_{\delta}(u, v)\right| \perp|\neg \alpha|(\alpha \vee \beta)$.

Definition 2.14. (Standard translation of terms)

If $u$ is a first-order variable and $A$ is a term then the corresponding first-order formula $S T(u, A)$ in $\mathcal{L}^{1}(B V)$ is inductively defined as follows:

- $S T\left(u, p_{n}\right)=P_{n}(u)$,

- $S T(u, f)=\perp$,

- $S T(u, t)=\mathrm{T}$,

- $S T(u,-A)=\neg S T(u, A)$,

- $S T(u, A \cup B)=S T(u, A) \vee S T(u, B)$ and

- $S T(u, A \cap B)=S T(u, A) \wedge S T(u, B)$. 


\section{Definition 2.15. (Standard translation of formulas)}

If $\phi$ is a formula then the corresponding first-order sentence $S T(\phi)$ in $\mathcal{L}^{1}(B V)$ is inductively defined as follows:

- $S T(A \equiv B)=\forall u(S T(u, A) \leftrightarrow S T(u, B))$,

- $S T(A \delta B)=\exists u\left(S T(u, A) \wedge \exists v\left(R_{\delta}(u, v) \wedge S T(v, B)\right)\right)$,

- $S T(\perp)=\perp$,

- $S T(\top)=\top$,

- $S T(\neg \phi)=\neg S T(\phi)$,

- $S T(\phi \vee \psi)=S T(\phi) \vee S T(\psi)$ and

- $S T(\phi \wedge \psi)=S T(\phi) \wedge S T(\psi)$.

Proposition 2.16. Let $\mathcal{M}=(W, R, V)$ be a model. For all terms $A$, for all $x \in W$ and for all formulas $\phi$,

- $x \in(A)^{V}$ iff $\mathcal{M} \models S T(u, A)[x]$ and

- $\mathcal{M} \models \phi$ iff $\mathcal{M} \models S T(\phi)$.

\section{Proof:}

The first item follows by induction on $A$ and the second one follows by induction on $\phi$.

The decidability of the 2-variable fragment of any first-order language with equality has been obtained by Mortimer [23]. The membership in NEXPTIME of its satisfiability problem has been established by Grädel et al. [20]. Hence, the embedding of our language into $\mathcal{L}^{1}(B V)$ considered in Proposition 2.16 implies that if $\mathcal{C}$ is a class of frames definable by a first-order sentence with at most 2 variables then the following decision problem is decidable in nondeterministic exponential time:

input: a formula $\phi$,

output: determine whether $\operatorname{sat}(\mathcal{C}, \phi)$.

\section{Bounded morphisms and bisimulations}

We recall the definitions of two relations between models presented in [5]: bounded morphisms and bisimulations. We will see that the satisfiability of formulas is invariant under these two relations.

\subsection{Bounded morphisms}

We first define bounded morphisms for our language.

\section{Definition 3.1. (Bounded morphisms)}

Let $\mathcal{M}=(W, R, V)$ and $\mathcal{M}^{\prime}=\left(W^{\prime}, R^{\prime}, V^{\prime}\right)$ be models. A bounded morphism from $\mathcal{M}$ to $\mathcal{M}^{\prime}$ is a surjection $f$ from $W$ to $W^{\prime}$ such that (i) for all $x, y \in W$, if $x R y$ then $f(x) R^{\prime} f(y)$, (ii) for all $x^{\prime}, y^{\prime} \in W^{\prime}$, if $x^{\prime} R^{\prime} y^{\prime}$ then there exists $x, y \in W$ such that $f(x)=x^{\prime}, f(y)=y^{\prime}$ and $x R y$ and (iii) for all Boolean variables $p$ and for all $x \in W, x \in V(p)$ iff $f(x) \in V^{\prime}(p)$. If there exists a bounded morphism from $\mathcal{M}$ to $\mathcal{M}^{\prime}$ then we say that $\mathcal{M}^{\prime}$ is a bounded morphic image of $\mathcal{M}$. 
Proposition 3.2. Let $\mathcal{M}=(W, R, V)$ and $\mathcal{M}^{\prime}=\left(W^{\prime}, R^{\prime}, V^{\prime}\right)$ be models. If $\mathcal{M}^{\prime}$ is a bounded morphic image of $\mathcal{M}$ then for all terms $A$, for all $x \in W$ and for all formulas $\phi$,

- $x \in(A)^{V}$ iff $f(x) \in(A)^{V^{\prime}}$ and

- $\mathcal{M} \models \phi$ iff $\mathcal{M}^{\prime} \models \phi$.

\section{Proof:}

The first item follows by induction on $A$ and the second one follows by induction on $\phi$.

By Proposition 3.2, we know that every model is modally equivalent to all its bounded morphic images. Is the converse true? That is, if two models are modally equivalent, must one of them be a bounded morphic image of the other? The answer is "no".

Example 3.3. Take the case of the models $\mathcal{M}_{\text {fin }}$ and $\mathcal{M}_{\text {inf }}$ defined in Example 2.13. As the reader is asked to show, $\mathcal{M}_{\text {fin }}$ and $\mathcal{M}_{\text {inf }}$ are modally equivalent but neither is $\mathcal{M}_{\text {fin }}$ a bounded morphic image of $\mathcal{M}_{\text {inf }}$ nor is $\mathcal{M}_{\text {inf }}$ a bounded morphic image of $\mathcal{M}_{\text {fin }}$.

\subsection{Bisimulations}

We now define bisimulations for our language.

\section{Definition 3.4. (Bisimulations)}

Let $\mathcal{M}=(W, R, V)$ and $\mathcal{M}^{\prime}=\left(W^{\prime}, R^{\prime}, V^{\prime}\right)$ be models. A bisimulation between $\mathcal{M}$ and $\mathcal{M}^{\prime}$ is a binary relation $Z$ between $W$ and $W^{\prime}$ such that (i) for all $x \in W$, there exists $x^{\prime} \in W^{\prime}$ such that $x Z x^{\prime}$, (ii) for all $x^{\prime} \in W^{\prime}$, there exists $x \in W$ such that $x Z x^{\prime}$, (iii) for all $x, y \in W$, if $x R y$ then there exists $x^{\prime}, y^{\prime} \in W^{\prime}$ such that $x Z x^{\prime}, y Z y^{\prime}$ and $x^{\prime} R^{\prime} y^{\prime}$, (iv) for all $x^{\prime}, y^{\prime} \in W^{\prime}$, if $x^{\prime} R^{\prime} y^{\prime}$ then there exists $x, y \in W$ such that $x Z x^{\prime}, y Z y^{\prime}$ and $x R y$ and (v) for all Boolean variables $p$, for all $x \in W$ and for all $x^{\prime} \in W^{\prime}$ such that $x Z x^{\prime}, x \in V(p)$ iff $x^{\prime} \in V^{\prime}(p)$. If there exists a bisimulation between $\mathcal{M}$ and $\mathcal{M}^{\prime}$ then we say that $\mathcal{M}$ and $\mathcal{M}^{\prime}$ are bisimilar.

We first prove a simple result.

Proposition 3.5. Let $\mathcal{M}=(W, R, V)$ and $\mathcal{M}^{\prime}=\left(W^{\prime}, R^{\prime}, V^{\prime}\right)$ be models. If the binary relation $Z$ between $W$ and $W^{\prime}$ is a bisimulation between $\mathcal{M}$ and $\mathcal{M}^{\prime}$ then for all terms $A$, for all $x \in W$, for all $x^{\prime} \in W^{\prime}$ and for all formulas $\phi$,

- if $x Z x^{\prime}$ then $x \in(A)^{V}$ iff $x^{\prime} \in(A)^{V^{\prime}}$ and

- $\mathcal{M} \models \phi$ iff $\mathcal{M}^{\prime} \models \phi$.

Proof:

The first item follows by induction on $A$ and the second one follows by induction on $\phi$.

The relation of the previous section, bounded morphism, is a bisimulation.

Proposition 3.6. Let $\mathcal{M}=(W, R, V)$ and $\mathcal{M}^{\prime}=\left(W^{\prime}, R^{\prime}, V^{\prime}\right)$ be models. If $\mathcal{M}^{\prime}$ is a bounded morphic image of $\mathcal{M}$ then $\mathcal{M}$ and $\mathcal{M}^{\prime}$ are bisimilar. 


\section{Proof:}

Let $f$ be a bounded morphism from $\mathcal{M}$ to $\mathcal{M}^{\prime}$. Let $Z$ be the binary relation between $W$ and $W^{\prime}$ such that for all $x \in W$ and for all $x^{\prime} \in W^{\prime}, x Z x^{\prime}$ iff $f(x)=x^{\prime}$. As the reader is asked to show, $Z$ is a bisimulation between $\mathcal{M}$ and $\mathcal{M}^{\prime}$.

By Proposition 3.5, we know that bisimilar models are modally equivalent. Is the converse true? That is, if two models are modally equivalent, must they be bisimilar? The answer is "no". Take the case of the models $\mathcal{M}_{\text {fin }}$ and $\mathcal{M}_{\text {inf }}$ defined in Example 2.13. As the reader is asked to show, $\mathcal{M}_{\text {fin }}$ and $\mathcal{M}_{\text {inf }}$ are modally equivalent but $\mathcal{M}_{\text {fin }}$ and $\mathcal{M}_{\text {inf }}$ are not bisimilar.

\subsection{Bounded morphisms, bisimulations and modal equivalence}

It is not possible to prove the converse to Proposition 3.2 in the case of finite models.

Example 3.7. To illustrate the truth of this, one has only to consider the finite models $\mathcal{M}=(W, R, V)$ and $\mathcal{M}^{\prime}=\left(W^{\prime}, R^{\prime}, V^{\prime}\right)$ defined as follows: $W=\{1,2,3\} ; R=\emptyset ; V(p)=\{1\}$ and $V(q)=\{2,3\}$; $W^{\prime}=\{1,2,3\} ; R^{\prime}=\emptyset ; V^{\prime}(p)=\{1,2\}$ and $V^{\prime}(q)=\{3\}$. As the reader is asked to show, $\mathcal{M}$ and $\mathcal{M}^{\prime}$ are modally equivalent but neither is $\mathcal{M}$ a bounded morphic image of $\mathcal{M}^{\prime}$ nor is $\mathcal{M}^{\prime}$ a bounded morphic image of $\mathcal{M}$.

Nevertheless, it is possible to prove the converse to Proposition 3.5 in the case of finite models. The next proposition is about an analogue of the Hennessy-Milner theorem in modal logic.

Proposition 3.8. Let $\mathcal{M}=(W, R, V)$ and $\mathcal{M}^{\prime}=\left(W^{\prime}, R^{\prime}, V^{\prime}\right)$ be finite models. If $\mathcal{M}$ and $\mathcal{M}^{\prime}$ are modally equivalent then $\mathcal{M}$ and $\mathcal{M}^{\prime}$ are bisimilar.

\section{Proof:}

Let $Z$ be the binary relation between $W$ and $W^{\prime}$ such that for all $x \in W$ and for all $x^{\prime} \in W^{\prime}, x Z x^{\prime}$ iff for all Boolean variables $p, x \in V(p)$ iff $x^{\prime} \in V^{\prime}(p)$. Let us show that $Z$ is a bisimulation between $\mathcal{M}$ and $\mathcal{M}^{\prime}$.

Let $x \in W$. Consider an enumeration $A_{0}, A_{1}, \ldots$ of all terms $A$ such that $x \in(A)^{V}$. Hence, for all non-negative integers $n, x \in\left(A_{0} \cap \ldots \cap A_{n}\right)^{V}$. Therefore, for all non-negative integers $n, \mathcal{M}^{\prime} \models$ $A_{0} \cap \ldots \cap A_{n} \not \equiv f$. Consequently, for all non-negative integers $n$, there exists $x_{n}^{\prime} \in W^{\prime}$ such that $x_{n}^{\prime} \in\left(A_{0}\right)^{V^{\prime}} \cap \ldots \cap\left(A_{n}\right)^{V^{\prime}}$. Since $W^{\prime}$ is finite, there exists $x^{\prime} \in W^{\prime}$ such that for all non-negative integers $n, x^{\prime} \in\left(A_{0}\right)^{V^{\prime}} \cap \ldots \cap\left(A_{n}\right)^{V^{\prime}}$. As the reader is asked to show, $x Z x^{\prime}$.

The second condition of bisimulations may be checked in a similar way.

Let $x \in W$ and $y \in W$ be such that $x R y$. Consider an enumeration $A_{0}, A_{1}, \ldots$ of all terms $A$ such that $x \in(A)^{V}$ and an enumeration $B_{0}, B_{1}, \ldots$ of all terms $B$ such that $y \in(B)^{V}$. Hence, for all non-negative integers $n, x \in\left(A_{0} \cap \ldots \cap A_{n}\right)^{V}$ and $y \in\left(B_{0} \cap \ldots \cap B_{n}\right)^{V}$. Therefore, for all non-negative integers $n, \mathcal{M}^{\prime} \models\left(A_{0} \cap \ldots \cap A_{n}\right) \delta\left(B_{0} \cap \ldots \cap B_{n}\right)$. Consequently, for all nonnegative integers $n$, there exists $x_{n}^{\prime}, y_{n}^{\prime} \in W^{\prime}$ such that $x_{n}^{\prime} R^{\prime} y_{n}^{\prime}, x_{n}^{\prime} \in\left(A_{0}\right)^{V^{\prime}} \cap \ldots \cap\left(A_{n}\right)^{V^{\prime}}$ and $y_{n}^{\prime} \in\left(B_{0}\right)^{V^{\prime}} \cap \ldots \cap\left(B_{n}\right)^{V^{\prime}}$. Since $W^{\prime}$ is finite, there exists $x^{\prime}, y^{\prime} \in W^{\prime}$ such that $x^{\prime} R^{\prime} y^{\prime}$ and for all non-negative integers $n, x^{\prime} \in\left(A_{0}\right)^{V^{\prime}} \cap \ldots \cap\left(A_{n}\right)^{V^{\prime}}$ and $y^{\prime} \in\left(B_{0}\right)^{V^{\prime}} \cap \ldots \cap\left(B_{n}\right)^{V^{\prime}}$. As the reader is asked to show, $x Z x^{\prime}$ and $y Z y^{\prime}$.

The fourth condition of bisimulations may be checked in a similar way.

The fifth condition of bisimulations is immediate. 


\section{Modal definability and modal undefinability}

In the setting of equivalence relations, modal definability and modal undefinability of first-order definable classes of frames have been investigated by Balbiani and Tinchev [2]. In the general setting, we study below the modal definability and the modal undefinability of several classes of frames.

\subsection{Preliminary definitions}

\section{Definition 4.1. (Modal definability)}

Let $\mathcal{C}$ be a class of frames. We shall say that $\mathcal{C}$ is modally definable by the formula $\phi$ iff for all frames $\mathcal{F}$, $\mathcal{F}$ is in $\mathcal{C}$ iff $\operatorname{val}(\mathcal{F}, \phi) . \mathcal{C}$ is said to be modally definable by a formula iff there exists a formula $\phi$ such that $\mathcal{C}$ is modally definable by $\phi$. We shall say that $\mathcal{C}$ is modally definable by the set $\Gamma$ of formulas iff for all frames $\mathcal{F}, \mathcal{F}$ is in $\mathcal{C}$ iff $\operatorname{val}(\mathcal{F}, \Gamma) . \mathcal{C}$ is said to be modally definable by a set of formulas iff there exists a set $\Gamma$ of formulas such that $\mathcal{C}$ is modally definable by $\Gamma$.

\subsection{Modal definability}

Definition 4.2. (Reflexivity, seriality, density, etc)

Let $\mathcal{F}=(W, R)$ be a frame. We shall say that (i) $\mathcal{F}$ is reflexive iff for all $x \in W, x R x$, (ii) $\mathcal{F}$ is serial iff for all $x \in W$, there exists $y \in W$ such that $x R y$, (iii) $\mathcal{F}$ is dense iff for all $x, y \in W$, if $x R y$ then there exists $z \in W$ such that $x R z$ and $z R y$, (iv) $\mathcal{F}$ is connected iff for all $x, y \in W$, if $x \neq y$ then there exists a positive integer $N$ and there exists a sequence $\left(z_{0}, \ldots, z_{N}\right)$ in $W$ such that $z_{0}=x, z_{N}=y$ and for all positive integers $k$, if $k \leq N$ then $z_{k-1} R z_{k}$, (v) $\mathcal{F}$ is non-2-colourable iff possible worlds in $W$ cannot be coloured by colours from a given set of 2 colours such that each two possible worlds connected by $R$ have different colours and (vi) $\mathcal{F}$ is looping iff for all $x \in W$, there exists a positive integer $N$ and there exists a sequence $\left(y_{0}, \ldots, y_{N}\right)$ in $W$ such that $y_{0}=x, y_{N}=x$ and for all positive integers $k$, if $k \leq N$ then $y_{k-1} R y_{k}$.

Remark that properties (i), (ii) and (iii) are first-order definable whereas properties (iv), (v) and (vi) are not first-order definable. Note also that properties (i), (ii) and (iii) are modally definable in the ordinary language of modal logic ( $\square p \rightarrow p, \square p \rightarrow \diamond p, \square \square p \rightarrow \square p$ ) whereas properties (iv), (v) and (vi) are not modally definable in the ordinary language of modal logic.

Proposition 4.3. The following classes of frames are modally definable by the associated formulas: (i) the class of all reflexive frames $(p \not \equiv f \rightarrow p \delta p)$, (ii) the class of all serial frames $(p \not \equiv f \rightarrow p \delta t)$, (iii) the class of all dense frames $(p \delta q \rightarrow p \delta r \vee-r \delta q)$, (iv) the class of all connected frames $(p \not \equiv f \wedge-p \not \equiv$ $f \rightarrow p \delta-p)$, (v) the class of all non-2-colourable frames $((p \cup q) \equiv t \wedge(p \cap q) \equiv f \rightarrow p \delta p \vee q \delta q)$ and (vi) the class of all looping frames $((p \cap-q) \not \equiv f \rightarrow p \delta-q \vee q \delta-q)$.

\section{Proof:}

See $[3,4,5]$ for that part of the proof concerning reflexivity, seriality, density, connectedness and non-2colourability.

Let $\mathcal{F}=(W, R)$ be a frame. Suppose $\mathcal{F}$ is looping. Hence, for all $x \in W$, there exists a positive integer $N$ and there exists a sequence $\left(y_{0}, \ldots, y_{N}\right)$ in $W$ such that $y_{0}=x, y_{N}=x$ and for all positive integers $k$, if $k \leq N$ then $y_{k-1} R y_{k}$. Let $V$ be a valuation based on $\mathcal{F}$. The reader may easily verify that $(W, R, V) \models(p \cap-q) \not \equiv f \rightarrow p \delta-q \vee q \delta-q$. 
Let $\mathcal{F}=(W, R)$ be a frame. Suppose $\mathcal{F}$ is not looping. Hence, there exists $x \in W$ such that for all positive integers $N$ and for all sequences $\left(y_{0}, \ldots, y_{N}\right)$ in $W$, if $y_{0}=x$ and $y_{N}=x$ then there exists a positive integer $k$ such that $k \leq N$ and not $y_{k-1} R y_{k}$. Let $V$ be the valuation based on $\mathcal{F}$ defined as follows: $V(p)=R^{\star}(x)$ and $V(q)=R^{+}(x)$. The reader may easily verify that $(W, R, V) \not \models(p \cap-q) \not \equiv$ $f \rightarrow p \delta-q \vee q \delta-q$.

\subsection{Modal undefinability}

\section{Definition 4.4. (Next-reflexivity, transitivity, irreflexivity, etc)}

Let $\mathcal{F}=(W, R)$ be a frame. We shall say that (i) $\mathcal{F}$ is next-reflexive iff for all $x \in W$, there exists $y \in W$ such that $x R y$ and $y R y$, (ii) $\mathcal{F}$ is transitive iff for all $x, y \in W$, if there exists $z \in W$ such that $x R z$ and $z R y$ then $x R y$, (iii) $\mathcal{F}$ is irreflexive iff for all $x \in W$, not $x R x$, (iv) $\mathcal{F}$ is Church-Rosser iff for all $x, y, z \in W$, if $x R y$ and $x R z$ then there exists $t \in W$ such that $y R t$ and $z R t,(\mathbf{v}) \mathcal{F}$ is McKinsey iff for all subsets $X$ of $W$ and for all $x \in W$, there exists $y \in W$ such that $x R y$ and $R(y) \subseteq X$ or $R(y) \cap X=\emptyset$, (vi) $\mathcal{F}$ is converse well-founded iff for all infinite sequences $\left(x_{0}, x_{1}, \ldots\right)$ in $W$, there exists a positive integer $k$ such that not $x_{k-1} R x_{k}$, (vii) $\mathcal{F}$ is 2 -colourable iff possible worlds in $W$ can be coloured by colours from a given set of 2 colours such that each two possible worlds connected by $R$ have different colours and (viii) $\mathcal{F}$ is non-Hamiltonian iff for all positive integers $N$ and for all sequences $\left(x_{0}, \ldots, x_{N}\right)$ in $W$, if $x_{0}=x_{N}$ then there exists $x \in W$ such that $\operatorname{card}(\{k: k$ is a positive integer such that $k \leq N$ and $\left.\left.x=x_{k-1}\right\}\right) \neq 1$.

Remark that properties (i), (ii), (iii) and (iv) are first-order definable whereas properties (v), (vi), (vii) and (viii) are not first-order definable. Note also that properties (ii), (iv) and (v) are modally definable in the ordinary language of modal logic ( $\square p \rightarrow \square \square p, \diamond \square p \rightarrow \square \diamond p, \square \diamond p \rightarrow \diamond \square p$ ) whereas properties (i), (iii), (vi), (vii) and (viii) are not modally definable in the ordinary language of modal logic.

Proposition 4.5. The following classes of frames are not modally definable by a set of formulas: (i) the class of all next-reflexive frames, (ii) the class of all transitive frames, (iii) the class of all irreflexive frames, (iv) the class of all Church-Rosser frames, (v) the class of all McKinsey frames, (vi) the class of all converse well-founded frames, (vii) the class of all 2-colourable frames and (viii) the class of all non-Hamiltonian frames.

\section{Proof:}

(i) Suppose the class of all next-reflexive frames is modally definable by a set of formulas. Hence, there exists a set $\Gamma$ of formulas such that for all frames $\mathcal{F}, \mathcal{F}$ is next-reflexive iff $\operatorname{val}(\mathcal{F}, \Gamma)$. Let $\mathcal{F}=(W, R)$ and $\mathcal{F}^{\prime}=\left(W^{\prime}, R^{\prime}\right)$ be the frames defined as follows: $W=\mathbb{N} \cup\{\omega\} ; R=\{(i, j): i, j \in \mathbb{N} \cup\{\omega\}$ are such that $i \neq j$ or $i=\omega$ and $j=\omega\} ; W^{\prime}=\mathbb{N} \cup\left\{\omega_{1}, \omega_{2}\right\} ; R^{\prime}=\left\{(i, j): i, j \in \mathbb{N} \cup\left\{\omega_{1}, \omega_{2}\right\}\right.$ are such that $i \neq j\}$. Obviously, $\mathcal{F}$ is next-reflexive and $\mathcal{F}^{\prime}$ is not next-reflexive. Therefore, $\operatorname{val}(\mathcal{F}, \Gamma)$ and not $\operatorname{val}\left(\mathcal{F}^{\prime}, \Gamma\right)$. Since not $\operatorname{val}\left(\mathcal{F}^{\prime}, \Gamma\right)$, there exists a model $\mathcal{M}^{\prime}=\left(W^{\prime}, R^{\prime}, V^{\prime}\right)$ based on $\mathcal{F}^{\prime}$ such that $\mathcal{M}^{\prime} \not \models \phi$ for some formula $\phi$ such that $\phi \in \Gamma$. Since $W^{\prime}$ is infinite and $F V(\phi)$ is finite, there exists $i_{1}, i_{2} \in W^{\prime}$ such that $i_{1} \neq i_{2}$ and for all Boolean variables $p \in F V(\phi), i_{1} \in V^{\prime}(p)$ iff $i_{2} \in V^{\prime}(p)$. Without loss of generality, let us assume that $i_{1}=\omega_{1}$ and $i_{2}=\omega_{2}$. Let $\mathcal{M}=(W, R, V)$ be a model based on $\mathcal{F}$ such that for all Boolean variables $p \in F V(\phi)$, if $V^{\prime}(p) \subseteq \mathbb{N}$ then $V(p)=V^{\prime}(p)$ else $V(p)=\left(V^{\prime}(p) \cap \mathbb{N}\right) \cup\{\omega\}$. Since $\operatorname{val}(\mathcal{F}, \Gamma)$ and $\phi \in \Gamma, \mathcal{M} \models \phi$. Now, we consider the binary relation $Z$ between $W$ and $W^{\prime}$ defined as follows: $Z=\{(i, i): i \in \mathbb{N}\} \cup\left\{\left(\omega, \omega_{1}\right),\left(\omega, \omega_{2}\right)\right\}$. The reader 
may easily verify that $Z$ is a bisimulation between $\mathcal{M}$ and $\mathcal{M}^{\prime}$ if one restricts the language to $F V(\phi)$. Consequently, by Proposition 3.5, $\mathcal{M} \forall \phi$ or $\mathcal{M}^{\prime} \models \phi$ : a contradiction.

(ii) Suppose the class of all transitive frames is modally definable by a set of formulas. Hence, there exists a set $\Gamma$ of formulas such that for all frames $\mathcal{F}, \mathcal{F}$ is transitive iff $\operatorname{val}(\mathcal{F}, \Gamma)$. Let $\mathcal{F}=(W, R)$

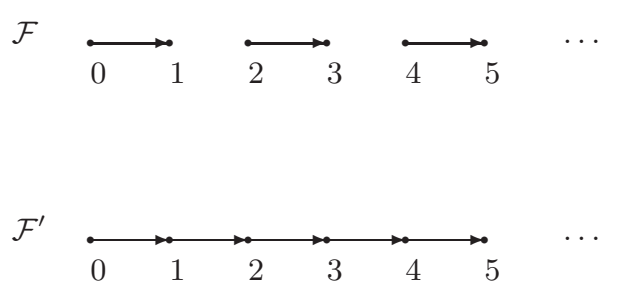

Figure 1.

and $\mathcal{F}^{\prime}=\left(W^{\prime}, R^{\prime}\right)$ be the frames defined as follows (see also Figure 1): $W=\mathbb{N} ; R=\{(2 i, 2 i+1)$ : $i \in \mathbb{N}\} ; W^{\prime}=\mathbb{N} ; R^{\prime}=\{(i, i+1): i \in \mathbb{N}\}$. Obviously, $\mathcal{F}$ is transitive and $\mathcal{F}^{\prime}$ is not transitive. Therefore, $\operatorname{val}(\mathcal{F}, \Gamma)$ and not $\operatorname{val}\left(\mathcal{F}^{\prime}, \Gamma\right)$. Let $f$ be the surjection from $W$ to $W^{\prime}$ defined as follows: $f(2 i)=i$ and $f(2 i+1)=i+1$. The reader may easily verify that $f$ is a bounded morphism from $\mathcal{F}$ to $\mathcal{F}^{\prime}$. Consequently, by Proposition 3.2, not $\operatorname{val}(\mathcal{F}, \Gamma)$ or $\operatorname{val}\left(\mathcal{F}^{\prime}, \Gamma\right)$ : a contradiction.

(iii) The argument concerning the class of all irreflexive frames is similar. It suffices to consider the

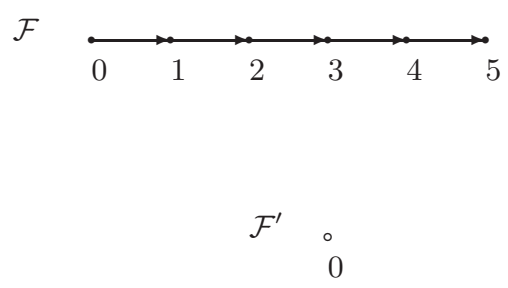

Figure 2.

frames $\mathcal{F}=(W, R)$ and $\mathcal{F}^{\prime}=\left(W^{\prime}, R^{\prime}\right)$ defined as follows (see also Figure 2$): W=\mathbb{N} ; R=\{(i, i+1)$ : $i \in \mathbb{N}\} ; W^{\prime}=\{0\} ; R^{\prime}=\{(0,0)\}$; together with the surjection $f$ from $W$ to $W^{\prime}$ defined as follows: $f(i)=0$.

(iv) The argument concerning the class of all Church-Rosser frames is similar. It suffices to consider the frames $\mathcal{F}=(W, R)$ and $\mathcal{F}^{\prime}=\left(W^{\prime}, R^{\prime}\right)$ defined as follows: $W=\mathbb{N} ; R=\{(2 i, 2 i+1): i \in$ $\mathbb{N}\} \cup\{(2 i+1,2 i+1): i \in \mathbb{N}\} ; W^{\prime}=\mathbb{N} ; R^{\prime}=\{(0, i+1): i \in \mathbb{N}\} \cup\{(i+1, i+1): i \in \mathbb{N}\} ;$ together with the surjection $f$ from $W$ to $W^{\prime}$ defined as follows: $f(2 i)=0$ and $f(2 i+1)=i+1$.

(v) The argument concerning the class of all McKinsey frames is similar. It suffices to consider the frames $\mathcal{F}=(W, R)$ and $\mathcal{F}^{\prime}=\left(W^{\prime}, R^{\prime}\right)$ defined as follows (see also Figure 3): $W=\mathbb{N} ; R=\{(i, i)$ : $i \in \mathbb{N}\} \cup\{(2 i, 2 i+1): i \in \mathbb{N}\} ; W^{\prime}=\mathbb{N} ; R^{\prime}=\{(i, i): i \in \mathbb{N}\} \cup\{(i, i+1): i \in \mathbb{N}\} ;$ together with the surjection $f$ from $W$ to $W^{\prime}$ defined as follows: $f(2 i)=i$ and $f(2 i+1)=i+1$.

(vi) The argument concerning the class of all converse well-founded frames is similar. It suffices to consider the frames $\mathcal{F}=(W, R)$ and $\mathcal{F}^{\prime}=\left(W^{\prime}, R^{\prime}\right)$ defined as follows (see also Figure 1 ): $W=\mathbb{N}$; 


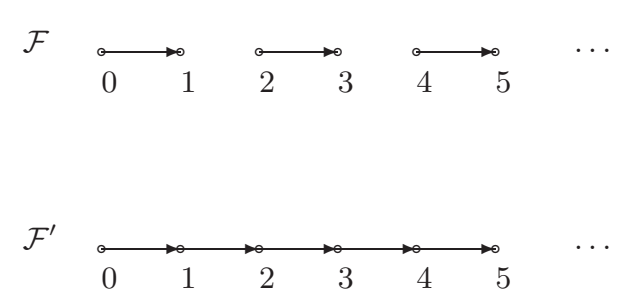

Figure 3.

$R=\{(2 i, 2 i+1): i \in \mathbb{N}\} ; W^{\prime}=\mathbb{N} ; R^{\prime}=\{(i, i+1): i \in \mathbb{N}\} ;$ together with the surjection $f$ from $W$ to $W^{\prime}$ defined as follows: $f(2 i)=i$ and $f(2 i+1)=i+1$.

(vii) The argument concerning the class of all 2-colourable frames is similar. It suffices to consider the frames $\mathcal{F}=(W, R)$ and $\mathcal{F}^{\prime}=\left(W^{\prime}, R^{\prime}\right)$ defined as follows: $W=\{0,1\} ; R=\{(0,1),(1,0)\}$; $W^{\prime}=\{0\} ; R^{\prime}=\{(0,0)\}$; together with the surjection $f$ from $W$ to $W^{\prime}$ defined as follows: $f(0)=0$ and $f(1)=0$.

(viii) The argument concerning the class of all non-Hamiltonian frames is similar. It suffices to consider the frames $\mathcal{F}=(W, R)$ and $\mathcal{F}^{\prime}=\left(W^{\prime}, R^{\prime}\right)$ defined as follows: $W=\mathbb{N} ; R=\{(i, i+1): i \in \mathbb{N}\}$; $W^{\prime}=\{0,1\} ; R^{\prime}=\{(0,1),(1,0)\}$; together with the surjection $f$ from $W$ to $W^{\prime}$ defined as follows: $f(i)=i \bmod 2$.

\section{Finite models}

This section introduces a variant of the technique of the filtration. This variant is used in next section for proving results about the canonical model. See $[3,4,5]$ for the proofs of the results Section 5.1 and Section 5.2 contain.

\subsection{Filtration models}

\section{Definition 5.1. (Filtrations)}

Let $\mathcal{M}=(W, R, V)$ be a model and $B V^{\prime} \subseteq B V$ be a set of Boolean variables. Let $\equiv_{B V^{\prime}}$ be the binary relation on $W$ such that for all $x, y \in W, x \equiv_{B V^{\prime}} y$ iff for all Boolean variables $p \in B V^{\prime}, x \in V(p)$ iff $y \in V(p)$. Remark that $\equiv_{B V^{\prime}}$ is an equivalence relation on $W$ such that for all $x, y \in W, x \equiv_{B V^{\prime}} y$ iff for all terms $A \in t\left(B V^{\prime}\right), x \in(A)^{V}$ iff $y \in(A)^{V}$. We denote the equivalence class of $x \in W$ with respect to $\equiv_{B V^{\prime}}$ by $|x|_{B V^{\prime}}$. If the model $\mathcal{M}^{\prime}=\left(W^{\prime}, R^{\prime}, V^{\prime}\right)$ is such that (i) $W^{\prime}=\left\{|x|_{B V^{\prime}}: x \in W\right\}$, (ii) for all $x, y \in W$, if $x R y$ then $|x|_{B V^{\prime}} R^{\prime}|y|_{B V^{\prime}}$, (iii) for all $x, y \in W$, if $|x|_{B V^{\prime}} R^{\prime}|y|_{B V^{\prime}}$ then for all terms $A, B \in t\left(B V^{\prime}\right)$, if $x \in(A)^{V}$ and $y \in(B)^{V}$ then $\mathcal{M} \models A \delta B$ and (iv) for all Boolean variables $p \in B V^{\prime}, V^{\prime}(p)=\left\{|x|_{B V^{\prime}}: x \in V(p)\right\}$ then we say that $\mathcal{M}^{\prime}$ is a filtration of $\mathcal{M}$ through $B V^{\prime}$.

Here, the first result is

Lemma 5.2. If $B V^{\prime}$ is finite then $\operatorname{car} d\left(\left\{|x|_{B V^{\prime}}: x \in W\right\}\right) \leq 2^{\operatorname{card}\left(B V^{\prime}\right)}$.

The next proposition duplicates the filtration theorem in modal logic. 
Proposition 5.3. Let $\mathcal{M}=(W, R, V)$ and $\mathcal{M}^{\prime}=\left(W^{\prime}, R^{\prime}, V^{\prime}\right)$ be models and $B V^{\prime} \subseteq B V$ be a set of Boolean variables. If $\mathcal{M}^{\prime}$ is a filtration of $\mathcal{M}$ through $B V^{\prime}$ then for all terms $A \in t\left(B V^{\prime}\right)$, for all $x \in W$ and for all formulas $\phi \in f\left(B V^{\prime}\right)$,

- $x \in(A)^{V}$ iff $|x|_{B V^{\prime}} \in(A)^{V^{\prime}}$ and

- $\mathcal{M} \models \phi$ iff $\mathcal{M}^{\prime} \models \phi$.

\subsection{Finest filtration and coarsest filtration}

\section{Definition 5.4. (Finest and coarsest filtrations)}

Let $\mathcal{M}=(W, R, V)$ be a model and $B V^{\prime} \subseteq B V$ be a set of Boolean variables. As the reader is asked to show, the model $\mathcal{M}^{f}=\left(W^{f}, R^{f}, V^{f}\right)$ defined as follows: $W^{f}=\left\{|x|_{B V^{\prime}}: x \in W\right\}$; for all $x, y \in W,|x|_{B V^{\prime}} R^{f}|y|_{B V^{\prime}}$ iff there exists $z, t \in W$ such that $x \equiv_{B V^{\prime}} z, y \equiv_{B V^{\prime}} t$ and $z R t$; for all Boolean variables $p \in B V^{\prime}, V^{f}(p)=\left\{|x|_{B V^{\prime}}: x \in V(p)\right\}$; and the model $\mathcal{M}^{c}=\left(W^{c}, R^{c}, V^{c}\right)$ defined as follows: $W^{c}=\left\{|x|_{B V^{\prime}}: x \in W\right\}$; for all $x, y \in W,|x|_{B V^{\prime}} R^{c}|y|_{B V^{\prime}}$ iff for all terms $A, B \in t\left(B V^{\prime}\right)$, if $x \in(A)^{V}$ and $y \in(B)^{V}$ then $\mathcal{M} \models A \delta B$; for all Boolean variables $p \in B V^{\prime}$, $V^{c}(p)=\left\{|x|_{B V^{\prime}}: x \in V(p)\right\}$; are filtrations of $\mathcal{M}$ through $B V^{\prime}$. We call $\mathcal{M}^{f}$ the finest filtration of $\mathcal{M}$ through $B V^{\prime}$ and $\mathcal{M}^{c}$ the coarsest filtration of $\mathcal{M}$ through $B V^{\prime}$.

Here, the first result is

Proposition 5.5. Let $\mathcal{M}=(W, R, V)$ and $\mathcal{M}^{\prime}=\left(W^{\prime}, R^{\prime}, V^{\prime}\right)$ be models and $B V^{\prime} \subseteq B V$ be a set of Boolean variables. If $\mathcal{M}^{\prime}$ is a filtration of $\mathcal{M}$ through $B V^{\prime}$ then for all $x, y \in W$,

- if $|x|_{B V^{\prime}} R^{f}|y|_{B V^{\prime}}$ then $|x|_{B V^{\prime}} R^{\prime}|y|_{B V^{\prime}}$ and

- if $|x|_{B V^{\prime}} R^{\prime}|y|_{B V^{\prime}}$ then $|x|_{B V^{\prime}} R^{c}|y|_{B V^{\prime}}$.

The next proposition is about an analogue of the finite model property in modal logic.

Proposition 5.6. Let $\phi$ be a formula. If $\phi$ is satisfiable then there exists a finite model $\mathcal{M}^{\prime}=\left(W^{\prime}, R^{\prime}, V^{\prime}\right)$ such that

- $\operatorname{card}\left(W^{\prime}\right) \leq 2^{\operatorname{card}(F V(\phi))}$ and

- $\mathcal{M}^{\prime} \models \phi$.

\subsection{New results about filtration models}

In addition to the above results about filtration models, we have the following new result.

Proposition 5.7. Let $\mathcal{M}=(W, R, V)$ be a model and $B V^{\prime} \subseteq B V$ be a set of Boolean variables. If $B V^{\prime}$ is finite then for all $x, y \in W,|x|_{B V^{\prime}} R^{f}|y|_{B V^{\prime}}$ iff $|x|_{B V^{\prime}} R^{c}|y|_{B V^{\prime}}$.

\section{Proof:}

Suppose $B V^{\prime}$ is finite. Let $x \in W$ and $y \in W$.

If $|x|_{B V^{\prime}} R^{f}|y|_{B V^{\prime}}$ then by Proposition 5.5, $|x|_{B V^{\prime}} R^{c}|y|_{B V^{\prime}}$.

If $|x|_{B V^{\prime}} R^{c}|y|_{B V^{\prime}}$ then for all terms $A, B \in t\left(B V^{\prime}\right)$, if $x \in(A)^{V}$ and $y \in(B)^{V}$ then $\mathcal{M} \models A \delta B$. 
Since $B V^{\prime}$ is finite, there exists a term $A_{x} \in t\left(B V^{\prime}\right)$ such that for all $z \in W, z \in\left(A_{x}\right)^{V}$ iff $x \equiv_{B V^{\prime}} z$ and there exists a term $B_{y} \in t\left(B V^{\prime}\right)$ such that for all $t \in W, t \in\left(B_{y}\right)^{V}$ iff $y \equiv_{B V^{\prime}} t$. Hence, $\mathcal{M} \models A_{x} \delta B_{y}$. Therefore, there exists $z, t \in W$ such that $z R t, z \in\left(A_{x}\right)^{V}$ and $t \in\left(B_{y}\right)^{V}$. Consequently, $|x|_{B V^{\prime}} R^{f}|y|_{B V^{\prime}}$

Hence, if $B V^{\prime}$ is finite then the finest filtration through $B V^{\prime}$ and the coarsest filtration through $B V^{\prime}$ coincide. Moreover,

Proposition 5.8. Let $\mathcal{M}=(W, R, V)$ be a model, $B V^{\prime} \subseteq B V$ be a set of Boolean variables and $B V^{\prime \prime} \subseteq B V$ be a set of Boolean variables. If $B V^{\prime}$ is finite and $B V^{\prime} \subseteq B V^{\prime \prime}$ then the filtration of $\mathcal{M}$ through $B V^{\prime}$ is a homomorphic image of any filtration of $\mathcal{M}$ through $B V^{\prime \prime}$.

Proof:

Suppose $B V^{\prime}$ is finite and $B V^{\prime} \subseteq B V^{\prime \prime}$. Let $\mathcal{M}^{B V^{\prime}}=\left(W^{B V^{\prime}}, R^{B V^{\prime}}, V^{B V^{\prime}}\right)$ be the filtration of $\mathcal{M}$ through $B V^{\prime}$ and $\mathcal{M}^{B V^{\prime \prime}}=\left(W^{B V^{\prime \prime}}, R^{B V^{\prime \prime}}, V^{B V^{\prime \prime}}\right)$ be any filtration of $\mathcal{M}$ through $B V^{\prime \prime}$. Let $f$ be the surjection from $W^{B V^{\prime \prime}}$ to $W^{B V^{\prime}}$ defined as follows: $f\left(|x|_{B V^{\prime \prime}}\right)=|x|_{B V^{\prime}}$. The reader may easily verify that $f$ is an homomorphism from $\mathcal{M}^{B V^{\prime \prime}}$ to $\mathcal{M}^{B V^{\prime}}$.

\section{Axiomatization and canonical model construction}

This section introduces a variant of the technique of the canonical model. This variant is used in next section for proving results about canonicity. See $[3,4,5]$ for the proofs of the results Section 6.2 and Section 6.3 contain.

\subsection{Axiomatization}

We first define the notion of logic for our language.

Definition 6.1. (Logics)

We shall say that a set of formulas is a logic iff it is closed under the following rules of inference:

- modus ponens: from $\phi$ and $\phi \rightarrow \psi$ infer $\psi$ and

- substitution: from $\phi$ infer $(\phi)^{\sigma}$,

it contains all instances of tautologies of the classical propositional logic, the theory of Boolean algebras - i.e. all instances of axioms for non-degenerate Boolean algebras in terms of $\equiv$ - and all instances of the following formulas:

- $A \delta B \rightarrow A \not \equiv f \wedge B \not \equiv f$

- $(A \cup B) \delta C \leftrightarrow A \delta C \vee B \delta C$ and

- $A \delta(B \cup C) \leftrightarrow A \delta B \vee A \delta C$.

We will use $L, M, N$, etc, for logics.

Remark that for all classes $\mathcal{C}$ of frames, $\operatorname{val}(\mathcal{C})$ is a logic. 


\section{Definition 6.2. (Classes of frames defined by logics)}

The class of (finite) frames defined by a logic $L$ is the class of all (finite) frames $\mathcal{F}$ such that for all formulas $\phi$, if $\phi \in L$ then $\operatorname{val}(\mathcal{F}, \phi)$.

Obviously, the set of all logics is a partially ordered set with respect to set inclusion.

\section{Definition 6.3. (Particular logics)}

Seeing that the intersection of any collection of logics is again a logic and the closure under modus ponens of the union of any collection of logics is again a logic, there exists a least logic, denoted $L_{\min }$, and there exists a greatest logic, denoted $L_{\max }$. Note that $L_{\max }$ is the set of all formulas. Of course, a logic $L$ is the set of all formulas iff there is a formula $\phi$ such that $\phi \in L$ and $\neg \phi \in L$ iff $\perp \in L$. A logic $L$ will be defined to be consistent iff $\perp \notin L$. For all formulas $\phi$, let $L_{\phi}$ be the least logic containing $\phi$.

\subsection{Canonical model}

Let $x$ be a set of terms. $x$ is said to be consistent iff for all non-negative integers $n$ and for all terms $A_{1}, \ldots, A_{n} \in x$, the formula $A_{1} \cap \ldots \cap A_{n} \equiv f$ is not derivable from the theory of Boolean algebras. We shall say that $x$ is maximal iff for all terms $A, A \in x$ or $-A \in x$. Let $L$ be a logic. We shall say that a set of formulas is an $L$-theory iff it is closed under the rule of modus ponens and it contains $L$. We will use $\Gamma, \Delta, \Lambda$, etc, for $L$-theories. For all sets $\Sigma$ of formulas, let $L+\Sigma$ be the set of all formulas $\phi$ such that there exists a non-negative integer $n$ and there exists formulas $\psi_{1}, \ldots, \psi_{n} \in \Sigma$ such that $\psi_{1} \wedge \ldots \wedge \psi_{n} \rightarrow \phi \in L$. Obviously, $L+\Sigma$ is the least $L$-theory containing $\Sigma$. Let us be clear that the set of all $L$-theories is a partially ordered set with respect to set inclusion. The least $L$-theory is $L$ and the greatest $L$-theory is the set of all formulas. Let $\Gamma$ be an $L$-theory. Of course, $\Gamma$ is the set of all formulas iff there is a formula $\phi$ such that $\phi \in \Gamma$ and $\neg \phi \in \Gamma$ iff $\perp \in \Gamma$. $\Gamma$ will be defined to be consistent iff $\perp \notin \Gamma$. We shall say that $\Gamma$ is maximal iff for all formulas $\phi, \phi \in \Gamma$ or $\neg \phi \in \Gamma$. Three lemmas support the technique of the canonical model for $L$ : the Lindenbaum's lemma, the diamond lemma and the truth lemma. The next lemma duplicates the Lindenbaum's lemma in modal logic.

Lemma 6.4. Let $\Gamma$ be a consistent $L$-theory. There exists a maximal consistent $L$-theory $\Delta$ such that $\Gamma \subseteq \Delta$.

The next lemma duplicates the diamond lemma in modal logic.

Lemma 6.5. Let $\Gamma$ be a maximal consistent $L$-theory. For all terms $A, B$,

- if $A \not \equiv f \in \Gamma$ then there exists a maximal consistent set $x$ of terms such that $A \in x$ and for all terms $A^{\prime}$, if $A^{\prime} \in x$ then $A^{\prime} \not \equiv f \in \Gamma$ and

- if $A \delta B \in \Gamma$ then there exists maximal consistent sets $x, y$ of terms such that $A \in x, B \in y$ and for all terms $A^{\prime}, B^{\prime}$, if $A^{\prime} \in x$ and $B^{\prime} \in y$ then $A^{\prime} \delta B^{\prime} \in \Gamma$.

Let $\Gamma$ be a maximal consistent $L$-theory. The canonical model for $\Gamma$ is the ordered triple $\mathcal{M}_{\Gamma}=$ $\left(W_{\Gamma}, R_{\Gamma}, V_{\Gamma}\right)$ where $W_{\Gamma}$ is the set of all maximal consistent sets $x$ of terms such that for all terms $A$, if $A \in x$ then $A \not \equiv f \in \Gamma ; R_{\Gamma}$ is the binary relation on $W_{\Gamma}$ such that $x R_{\Gamma} y$ iff for all terms $A, B$, if $A \in x$ and $B \in y$ then $A \delta B \in \Gamma ; V_{\Gamma}$ is the function assigning to each Boolean variable $p$ the subset $V_{\Gamma}(p)$ of $W_{\Gamma}$ such that $x \in V_{\Gamma}(p)$ iff $p \in x$. The pair $\mathcal{F}_{\Gamma}=\left(W_{\Gamma}, R_{\Gamma}\right)$ is called the canonical frame for $\Gamma$. The next lemma duplicates the truth lemma in modal logic. 
Lemma 6.6. For all terms $A$ and for all formulas $\phi$,

- $x \in(A)^{V_{\Gamma}}$ iff $A \in x$ and

- $\mathcal{M}_{\Gamma} \models \phi$ iff $\phi \in \Gamma$.

The next result says that the frames of the filtrations of $\mathcal{M}_{\Gamma}$ through finite sets of Boolean variables validate $L$.

Proposition 6.7. Let $\Gamma$ be a maximal consistent $L$-theory, $\mathcal{M}^{\prime}=\left(W^{\prime}, R^{\prime}, V^{\prime}\right)$ be a model and $B V^{\prime} \subseteq$ $B V$ be a set of Boolean variables. If $B V^{\prime}$ is finite and $\mathcal{M}^{\prime}$ is the filtration of $\mathcal{M}_{\Gamma}$ through $B V^{\prime}$ then $\operatorname{val}\left(\left(W^{\prime}, R^{\prime}\right), L\right)$.

\subsection{Completeness}

The key result concerning completeness is the following

Proposition 6.8. Let $\phi$ be a formula. If $\phi \notin L$ then there exists a finite frame $\mathcal{F}$ such that $\operatorname{val}(\mathcal{F}, L)$ and not $\operatorname{val}(\mathcal{F}, \phi)$.

By Proposition 6.8, it follows that every consistent logic is complete with respect to its class of finite frames. As a result,

Proposition 6.9. The logics obtained by adding to $L_{\min }$ the following formulas are complete with respect to the associated classes of frames: (i) $p \not \equiv f \rightarrow p \delta p$ (the class of all reflexive frames), (ii) $p \not \equiv f \rightarrow p \delta t$ (the class of all serial frames), (iii) $p \delta q \rightarrow p \delta r \vee-r \delta q$ (the class of all dense frames), (iv) $p \not \equiv f \wedge-p \not \equiv f \rightarrow p \delta-p$ (the class of all connected frames), (v) $(p \cup q) \equiv t \wedge(p \cap q) \equiv f \rightarrow p \delta p \vee q \delta q$ (the class of all non-2-colourable frames) and (vi) $(p \cap-q) \not \equiv f \rightarrow p \delta-q \vee q \delta-q$ (the class of all looping frames).

\section{Canonicity}

This section introduces and studies the concept of canonicity.

\subsection{Preliminary discussion}

Let $L$ be a logic. If $L$ is consistent then $\perp \notin L$. Hence, $L$ is a consistent $L$-theory. By Lemma 6.4, there exists a maximal consistent $L$-theory $\Gamma$ such that $L \subseteq \Gamma$. Let $\mathcal{M}_{\Gamma}=\left(W_{\Gamma}, R_{\Gamma}, V_{\Gamma}\right)$ be the canonical model for $\Gamma$ and $\mathcal{M}^{\prime}=\left(W^{\prime}, R^{\prime}, V^{\prime}\right)$ be the filtration of $\mathcal{M}_{\Gamma}$ through $\emptyset$. By Proposition 6.7, $\operatorname{val}\left(\left(W^{\prime}, R^{\prime}\right), L\right)$. Let $\Delta=\left\{\phi: \phi\right.$ is a formula such that $\left.\mathcal{M}^{\prime} \models \phi\right\}$. Obviously, $\Delta$ is a maximal consistent $L$-theory. Let $\mathcal{M}_{\Delta}=\left(W_{\Delta}, R_{\Delta}, V_{\Delta}\right)$ be the canonical model for $\Delta$. The reader may easily verify that $W^{\prime}$ contains exactly one possible world, say $x^{\prime}$, and $W_{\Delta}$ contains exactly one possible world, say $x_{\Delta}$. Moreover, $x^{\prime} R^{\prime} x^{\prime}$ iff $x_{\Delta} R_{\Delta} x_{\Delta}$. Consequently, $\left(W^{\prime}, R^{\prime}\right)$ is isomorphic to the canonical frame for $\Delta$. Hence, $\Delta$ is a maximal consistent $L$-theory such that $\left(W^{\prime}, R^{\prime}\right)$ is isomorphic to the canonical frame for $\Delta$. It follows immediately from the above discussion that for all consistent logics $L$, there exists a maximal consistent $L$-theory $\Gamma$ such that $\operatorname{val}\left(\mathcal{F}_{\Gamma}, L\right)$. 


\section{Definition 7.1. (Canonical logics)}

Let $L$ be a logic. $L$ is said to be canonical iff for all maximal consistent $L$-theories $\Gamma, \operatorname{val}\left(\mathcal{F}_{\Gamma}, L\right)$.

In order to prove the completeness of a logic with respect to the class of all its canonical frames, the concept of canonicity is essential. More precisely,

Proposition 7.2. Let $L$ be a logic. If $L$ is canonical then $L$ is complete with respect to the class of all its canonical frames.

\section{Proof:}

Suppose $L$ is canonical. Let $\phi$ be a formula such that $\phi \notin L$. By Lemma 6.4, there exists a maximal consistent $L$-theory $\Gamma$ such that $\phi \notin \Gamma$. Since $L$ is canonical, $\operatorname{val}\left(\mathcal{F}_{\Gamma}, L\right)$. Since $\phi \notin \Gamma$, by Lemma 6.6, not $\operatorname{val}\left(\mathcal{F}_{\Gamma}, \phi\right)$.

However, there are non-canonical logics. See Section 7.3 for examples of such non-canonical logics.

\subsection{Examples of canonical logics}

What about the concept of canonicity defined above? Let us try to develop some intuitions concerning it by considering a number of examples of canonical logics. Consider $L_{\min }$, the least logic. Since $L_{\min }$ is valid in all frames, $L_{\min }$ is canonical. In other respect,

Proposition 7.3. The following logics are canonical:

- $\operatorname{val}\left(\mathcal{C}_{r e f}\right)$ where $\mathcal{C}_{\text {ref }}$ is the class of all reflexive frames,

- $\operatorname{val}\left(\mathcal{C}_{\text {ser }}\right)$ where $\mathcal{C}_{\text {ser }}$ is the class of all serial frames and

- $\operatorname{val}\left(\mathcal{C}_{d e n}\right)$ where $\mathcal{C}_{d e n}$ is the class of all dense frames.

\section{Proof:}

Reflexive frames. Let $L_{r e f}$ be the logic obtained by adding to $L_{\text {min }}$ the formula $p \not \equiv f \rightarrow p \delta p$. By Proposition 6.9, $\operatorname{val}\left(\mathcal{C}_{r e f}\right)=L_{r e f}$. Let $\Gamma$ be a maximal consistent $L_{r e f}$-theory. By Proposition 4.3, it remains to show that the canonical frame $\mathcal{F}_{\Gamma}=\left(W_{\Gamma}, R_{\Gamma}\right)$ for $\Gamma$ is reflexive. Let $x \in W_{\Gamma}$. For all terms $A, B$, if $A \in x$ and $B \in x$ then $A \cap B \in x$. Hence, $(A \cap B) \not \equiv f \in \Gamma$. Therefore, using the axiom $(A \cap B) \not \equiv f \rightarrow(A \cap B) \delta(A \cap B),(A \cap B) \delta(A \cap B) \in \Gamma$. Consequently, $A \delta B \in \Gamma$. Thus, $x R_{\Gamma} x$.

Serial frames. Let $L_{s e r}$ be the logic obtained by adding to $L_{\min }$ the formula $p \not \equiv f \rightarrow p \delta t$. By Proposition 6.9, $\operatorname{val}\left(\mathcal{C}_{s e r}\right)=L_{\text {ser }}$. Let $\Gamma$ be a maximal consistent $L_{s e r}$-theory. By Proposition 4.3, it remains to show that the canonical frame $\mathcal{F}_{\Gamma}=\left(W_{\Gamma}, R_{\Gamma}\right)$ for $\Gamma$ is serial. Let $x \in W_{\Gamma}$. Consider an enumeration $A_{0}, A_{1}, \ldots$ of all terms $A$ such that $A \in x$ and an enumeration $B_{0}, B_{1}, \ldots$ of all terms. Hence, for all non-negative integers $n, A_{0} \cap \ldots \cap A_{n} \in x$. Therefore, $\left(A_{0} \cap \ldots \cap A_{n}\right) \not \equiv f \in \Gamma$. Consequently, using the axiom $\left(A_{0} \cap \ldots \cap A_{n}\right) \not \equiv f \rightarrow\left(A_{0} \cap \ldots \cap A_{n}\right) \delta t,\left(A_{0} \cap \ldots \cap A_{n}\right) \delta t \in \Gamma$. Thus, there exists a sequence $\left(\beta_{0}^{n}, \ldots, \beta_{n}^{n}\right)$ in $\{0,1\}^{\star}$ such that $\left(A_{0} \cap \ldots \cap A_{n}\right) \delta\left(B_{0}^{\beta_{0}^{n}} \cap \ldots \cap B_{n}^{\beta_{n}^{n}}\right) \in \Gamma$. By König's infinity lemma for trees, there exists a sequence $\left(\beta_{0}, \beta_{1}, \ldots\right)$ in $\{0,1\}^{\omega}$ such that for all nonnegative integers $n,\left(A_{0} \cap \ldots \cap A_{n}\right) \delta\left(B_{0}^{\beta_{0}} \cap \ldots \cap B_{n}^{\beta_{n}}\right) \in \Gamma$. Let $y=\left\{B_{0}^{\beta_{0}}, B_{1}^{\beta_{1}}, \ldots\right\}$. The reader may easily demonstrate that $y \in W_{\Gamma}$ and $x R_{\Gamma} y$. 
Dense frames. Let $L_{d e n}$ be the logic obtained by adding to $L_{\min }$ the formula $p \delta q \rightarrow p \delta r \vee-r \delta q$. By Proposition 6.9, $\operatorname{val}\left(\mathcal{C}_{d e n}\right)=L_{d e n}$. Let $\Gamma$ be a maximal consistent $L_{d e n}$-theory. By Proposition 4.3, it remains to show that the canonical frame $\mathcal{F}_{\Gamma}=\left(W_{\Gamma}, R_{\Gamma}\right)$ for $\Gamma$ is dense. Let $x \in W_{\Gamma}$ and $y \in$ $W_{\Gamma}$ be such that $x R_{\Gamma} y$. Consider an enumeration $A_{0}, A_{1}, \ldots$ of all terms $A$ such that $A \in x$, an enumeration $B_{0}, B_{1}, \ldots$ of all terms $B$ such that $B \in y$ and an enumeration $C_{0}, C_{1}, \ldots$ of all terms. Hence, for all non-negative integers $n, A_{0} \cap \ldots \cap A_{n} \in x$ and $B_{0} \cap \ldots \cap B_{n} \in y$. Therefore, $\left(A_{0} \cap\right.$ $\left.\ldots \cap A_{n}\right) \delta\left(B_{0} \cap \ldots \cap B_{n}\right) \in \Gamma$. Let $S^{0}$ be the set of all sequences $\left(\gamma_{0}^{n}, \ldots, \gamma_{n}^{n}\right)$ in $\{0,1\}^{\star}$ such that $\left(A_{0} \cap \ldots \cap A_{n}\right) \delta\left(C_{0}^{\gamma_{0}^{n}} \cap \ldots \cap C_{n}^{\gamma_{n}^{n}}\right) \notin \Gamma$ and $S^{1}$ be the set of all sequences $\left(\gamma_{0}^{n}, \ldots, \gamma_{n}^{n}\right)$ in $\{0,1\}^{\star}$ such that $\left(A_{0} \cap \ldots \cap A_{n}\right) \delta\left(C_{0}^{\gamma_{0}^{n}} \cap \ldots \cap C_{n}^{\gamma_{n}^{n}}\right) \in \Gamma$ where $C^{0}=-C$ and $C^{1}=C$ for every term $C$. As the reader is asked to show, $-\bigcup\left\{C_{0}^{\gamma_{0}^{n}} \cap \ldots \cap C_{n}^{\gamma_{n}^{n}}:\left(\gamma_{0}^{n}, \ldots, \gamma_{n}^{n}\right) \in S^{0}\right\} \delta\left(B_{0} \cap \ldots \cap B_{n}\right) \rightarrow \bigcup\left\{C_{0}^{\gamma_{0}^{n}} \cap \ldots \cap C_{n}^{\gamma_{n}^{n}}\right.$ : $\left.\left(\gamma_{0}^{n}, \ldots, \gamma_{n}^{n}\right) \in S^{1}\right\} \delta\left(B_{0} \cap \ldots \cap B_{n}\right) \in \Gamma$. Since $\left(A_{0} \cap \ldots \cap A_{n}\right) \delta\left(B_{0} \cap \ldots \cap B_{n}\right) \in \Gamma$, using the axiom $\left(A_{0} \cap \ldots \cap A_{n}\right) \delta\left(B_{0} \cap \ldots \cap B_{n}\right) \rightarrow\left(A_{0} \cap \ldots \cap A_{n}\right) \delta \bigcup\left\{C_{0}^{\gamma_{0}^{n}} \cap \ldots \cap C_{n}^{\gamma_{n}^{n}}:\left(\gamma_{0}^{n}, \ldots, \gamma_{n}^{n}\right) \in\right.$ $\left.S^{0}\right\} \vee-\bigcup\left\{C_{0}^{\gamma_{0}^{n}} \cap \ldots \cap C_{n}^{\gamma_{n}^{n}}:\left(\gamma_{0}^{n}, \ldots, \gamma_{n}^{n}\right) \in S^{0}\right\} \delta\left(B_{0} \cap \ldots \cap B_{n}\right),\left(A_{0} \cap \ldots \cap A_{n}\right) \delta \bigcup\left\{C_{0}^{\gamma_{0}^{n}} \cap \ldots \cap C_{n}^{\gamma_{n}^{n}}:\right.$ $\left.\left(\gamma_{0}^{n}, \ldots, \gamma_{n}^{n}\right) \in S^{0}\right\} \in \Gamma$ or $-\bigcup\left\{C_{0}^{\gamma_{0}^{n}} \cap \ldots \cap C_{n}^{\gamma_{n}^{n}}:\left(\gamma_{0}^{n}, \ldots, \gamma_{n}^{n}\right) \in S^{0}\right\} \delta\left(B_{0} \cap \ldots \cap B_{n}\right) \in \Gamma$. Obviously, $\left(A_{0} \cap \ldots \cap A_{n}\right) \delta \bigcup\left\{C_{0}^{\gamma_{0}^{n}} \cap \ldots \cap C_{n}^{\gamma_{n}^{n}}:\left(\gamma_{0}^{n}, \ldots, \gamma_{n}^{n}\right) \in S^{0}\right\} \notin \Gamma$. Consequently, $-\bigcup\left\{C_{0}^{\gamma_{0}^{n}} \cap \ldots \cap C_{n}^{\gamma_{n}^{n}}:\right.$ $\left.\left(\gamma_{0}^{n}, \ldots, \gamma_{n}^{n}\right) \in S^{0}\right\} \delta\left(B_{0} \cap \ldots \cap B_{n}\right) \in \Gamma$. Since $-\bigcup\left\{C_{0}^{\gamma_{0}^{n}} \cap \ldots \cap C_{n}^{\gamma_{n}^{n}}:\left(\gamma_{0}^{n}, \ldots, \gamma_{n}^{n}\right) \in S^{0}\right\} \delta\left(B_{0} \cap\right.$ $\left.\ldots \cap B_{n}\right) \rightarrow \bigcup\left\{C_{0}^{\gamma_{0}^{n}} \cap \ldots \cap C_{n}^{\gamma_{n}^{n}}:\left(\gamma_{0}^{n}, \ldots, \gamma_{n}^{n}\right) \in S^{1}\right\} \delta\left(B_{0} \cap \ldots \cap B_{n}\right) \in \Gamma, \bigcup\left\{C_{0}^{\gamma_{0}^{n}} \cap \ldots \cap C_{n}^{\gamma_{n}^{n}}:\right.$ $\left.\left(\gamma_{0}^{n}, \ldots, \gamma_{n}^{n}\right) \in S^{1}\right\} \delta\left(B_{0} \cap \ldots \cap B_{n}\right) \in \Gamma$. Thus, there exists a sequence $\left(\gamma_{0}^{n}, \ldots, \gamma_{n}^{n}\right)$ in $\{0,1\}^{\star}$ such that $\left(A_{0} \cap \ldots \cap A_{n}\right) \delta\left(C_{0}^{\gamma_{0}^{n}} \cap \ldots \cap C_{n}^{\gamma_{n}^{n}}\right) \in \Gamma$ and $\left(C_{0}^{\gamma_{0}^{n}} \cap \ldots \cap C_{n}^{\gamma_{n}^{n}}\right) \delta\left(B_{0} \cap \ldots \cap B_{n}\right) \in \Gamma$. By König's infinity lemma for trees, there exists a sequence $\left(\gamma_{0}, \gamma_{1}, \ldots\right)$ in $\{0,1\}^{\omega}$ such that for all non-negative integers $n,\left(A_{0} \cap \ldots \cap A_{n}\right) \delta\left(C_{0}^{\gamma_{0}} \cap \ldots \cap C_{n}^{\gamma_{n}}\right) \in \Gamma$ and $\left(C_{0}^{\gamma_{0}} \cap \ldots \cap C_{n}^{\gamma_{n}}\right) \delta\left(B_{0} \cap \ldots \cap B_{n}\right) \delta \in \Gamma$. Let $z=\left\{C_{0}^{\gamma_{0}}, C_{1}^{\gamma_{1}}, \ldots\right\}$. The reader may easily demonstrate that $z \in W_{\Gamma}, x R_{\Gamma} z$ and $z R_{\Gamma} y$.

We will see in Section 8 that Proposition 7.3 is an immediate consequence of the more general result stated in Proposition 8.2.

\subsection{Examples of non-canonical logics}

Now, we consider a number of examples of non-canonical logics. Let $p_{0}, p_{1}, \ldots$ be an enumeration of $B V$. For all non-negative integers $n$ and for all sequences $\vec{\alpha}=\left(\alpha_{0}, \ldots, \alpha_{n}\right)$ in $\{0,1\}^{\star}$, let $\tau(\vec{\alpha})=$ $p_{0}^{\alpha_{0}} \cap \ldots \cap p_{n}^{\alpha_{n}}$ where $p^{0}=-p$ and $p^{1}=p$ for every Boolean variable $p,|\vec{\alpha}|=n,\lfloor\vec{\alpha}\rfloor=\alpha_{0} \ldots \alpha_{n}$ and $\lceil\vec{\alpha}\rceil=\alpha_{n} \ldots \alpha_{0}$, i.e. the non-negative integers represented by $\alpha_{0}, \ldots, \alpha_{n}$ and $\alpha_{n}, \ldots, \alpha_{0}$ in the binary system.

Proposition 7.4. The following logic is not canonical:

- $\operatorname{val}\left(\mathcal{C}_{\text {con }}\right)$ where $\mathcal{C}_{\text {con }}$ is the class of all connected frames.

\section{Proof:}

Let $L_{c o n}$ be the logic obtained by adding to $L_{\text {min }}$ the formula $p \not \equiv f \wedge-p \not \equiv f \rightarrow p \delta-p$. By Proposition $6.9, \operatorname{val}\left(\mathcal{C}_{c o n}\right)=L_{c o n}$. Let $n$ be a non-negative integer. We consider the set $\Sigma_{n}$ of formulas defined as follows: 
- $\Sigma_{n}=\left\{\tau(\vec{\alpha}) \delta \tau(\vec{\beta}): \vec{\alpha}\right.$ is a sequence in $\{0,1\}^{\star}$ and $\vec{\beta}$ is a sequence in $\{0,1\}^{\star}$ such that $|\vec{\alpha}|=n$, $|\vec{\beta}|=n$ and $|\lfloor\vec{\alpha}\rfloor-\lfloor\vec{\beta}\rfloor| \leq 1\} \cup\left\{\tau(\vec{\alpha}) \bar{\delta} \tau(\vec{\beta}): \vec{\alpha}\right.$ is a sequence in $\{0,1\}^{\star}$ and $\vec{\beta}$ is a sequence in $\{0,1\}^{\star}$ such that $|\vec{\alpha}|=n,|\vec{\beta}|=n$ and $\left.|\lfloor\vec{\alpha}\rfloor-\lfloor\vec{\beta}\rfloor|>1\right\}$.

We consider the frame $\mathcal{F}_{n}=\left(W_{n}, R_{n}\right)$ defined as follows:

- $W_{n}$ is the set of all sequences $\vec{\alpha}$ in $\{0,1\}^{\star}$ such that $|\vec{\alpha}|=n$,

- $R_{n}$ is the binary relation on $W_{n}$ such that $\vec{\alpha} R_{n} \vec{\beta}$ iff $|\lfloor\vec{\alpha}\rfloor-\lfloor\vec{\beta}\rfloor| \leq 1$

and the valuation $V_{n}$ based on $\mathcal{F}_{n}$ defined as follows:

- $V_{n}$ is the function assigning to each Boolean variable $p$ the subset $V_{n}(p)$ of $W_{n}$ such that $\vec{\alpha} \in V_{n}(p)$ iff there exists a non-negative integer $k$ such that $k \leq n, p=p_{k}$ and $\alpha_{k}=1$.

Note that $W_{0}=\{(0),(1)\}, W_{1}=\{(0,0),(0,1),(1,0),(1,1)\}$ and $W_{2}=\{(0,0,0),(0,0,1),(0,1,0)$, $(0,1,1),(1,0,0),(1,0,1),(1,1,0),(1,1,1)\}$.

Lemma 7.5. $\operatorname{val}\left(\mathcal{F}_{n}, L_{c o n}\right)$.

\section{Proof:}

It suffices to remark that $\mathcal{F}_{n}$ is connected.

Lemma 7.6. $\left(\mathcal{F}_{n}, V_{n}\right) \models \bigcup\left\{\Sigma_{i}: i\right.$ is a non-negative integer such that $\left.i \leq n\right\}$.

\section{Proof:}

Consider a non-negative integer $i$ such that $i \leq n$.

Let $\tau(\vec{\alpha}) \delta \tau(\vec{\beta})$ be a formula in $\Sigma_{i}$. Hence, $\vec{\alpha}$ is a sequence in $\{0,1\}^{\star}$ and $\vec{\beta}$ is a sequence in $\{0,1\}^{\star}$ such that $|\vec{\alpha}|=i,|\vec{\beta}|=i$ and $|\lfloor\vec{\alpha}\rfloor-\lfloor\vec{\beta}\rfloor| \leq 1$. Therefore, there exists sequences $\overrightarrow{\alpha^{\prime}}, \overrightarrow{\beta^{\prime}}$ in $\{0,1\}^{\star}$ such that $\left|\overrightarrow{\alpha^{\prime}}\right|=n,\left|\overrightarrow{\beta^{\prime}}\right|=n,\left|\left\lfloor\overrightarrow{\alpha^{\prime}}\right\rfloor-\left\lfloor\overrightarrow{\beta^{\prime}}\right\rfloor\right| \leq 1,\left(\alpha_{0}^{\prime}, \ldots, \alpha_{i}^{\prime}\right)=\left(\alpha_{0}, \ldots, \alpha_{i}\right)$ and $\left(\beta_{0}^{\prime}, \ldots, \beta_{i}^{\prime}\right)=\left(\beta_{0}, \ldots, \beta_{i}\right)$. Thus, $\left(\mathcal{F}_{n}, V_{n}\right) \models \tau(\vec{\alpha}) \delta \tau(\vec{\beta})$.

Let $\tau(\vec{\alpha}) \bar{\delta} \tau(\vec{\beta})$ be a formula in $\Sigma_{i}$. Hence, $\vec{\alpha}$ is a sequence in $\{0,1\}^{\star}$ and $\vec{\beta}$ is a sequence in $\{0,1\}^{\star}$ such that $|\vec{\alpha}|=i, \mid \vec{\beta}\rfloor=i$ and $|\lfloor\vec{\alpha}\rfloor-\lfloor\vec{\beta}\rfloor|>1$. Therefore, for all sequences $\overrightarrow{\alpha^{\prime}}, \overrightarrow{\beta^{\prime}}$ in $\{0,1\}^{\star}$, if $\left|\overrightarrow{\alpha^{\prime}}\right|=n$, $\left|\overrightarrow{\beta^{\prime}}\right|=n$ and $\left|\left\lfloor\overrightarrow{\alpha^{\prime}}\right\rfloor-\left\lfloor\overrightarrow{\beta^{\prime}}\right\rfloor\right| \leq 1$ then $\left(\alpha_{0}^{\prime}, \ldots, \alpha_{i}^{\prime}\right) \neq\left(\alpha_{0}, \ldots, \alpha_{i}\right)$ or $\left(\beta_{0}^{\prime}, \ldots, \beta_{i}^{\prime}\right) \neq\left(\beta_{0}, \ldots, \beta_{i}\right)$. Thus, $\left(\mathcal{F}_{n}, V_{n}\right) \models \tau(\vec{\alpha}) \bar{\delta} \tau(\vec{\beta})$.

Let $\Sigma=\bigcup\left\{\Sigma_{n}: n\right.$ is a non-negative integer $\}$.

Lemma 7.7. $L_{c o n}+\Sigma$ is a consistent $L_{c o n}$-theory.

\section{Proof:}

By Lemma 7.5 and Lemma 7.6.

By Lemma 6.4, there exists a maximal consistent $L_{\text {con }}$-theory $\Delta$ such that $\Sigma \subseteq \Delta$. By Proposition 4.3, it remains to show that the canonical frame $\mathcal{F}_{\Delta}=\left(W_{\Delta}, R_{\Delta}\right)$ for $\Delta$ is not connected. Suppose $\mathcal{F}_{\Delta}$ is connected. The reader may easily verify that for all non-negative integers $n, p_{0}^{0} \cap \ldots \cap p_{n}^{0} \not \equiv f \in \Delta$ and $p_{0}^{1} \cap \ldots \cap p_{n}^{1} \not \equiv f \in \Delta$. Hence, there exists $x_{0} \in W_{\Delta}$ such that $\left\{p_{0}^{0}, p_{1}^{0}, \ldots\right\} \subseteq x_{0}$ and there exists 
$x_{1} \in W_{\Delta}$ such that $\left\{p_{0}^{1}, p_{1}^{1}, \ldots\right\} \subseteq x_{1}$. Obviously, $x_{0} \neq x_{1}$. Since $\mathcal{F}_{\Delta}$ is connected, there exists a positive integer $N$ and there exists a sequence $\left(z_{0}, \ldots, z_{N}\right)$ in $W_{\Delta}$ such that $z_{0}=x_{0}, z_{N}=x_{1}$ and for all positive integers $k$, if $k \leq N$ then $z_{k-1} R_{\Delta} z_{k}$. Let $n$ be a non-negative integer such that $2^{n+1}-1>N$ and $k$ be a non-negative integer such that $k \leq N$. Consider the sequence $\vec{\alpha}^{k}=\left(\alpha_{0}^{k}, \ldots, \alpha_{n}^{k}\right)$ in $\{0,1\}^{\star}$ such that for all non-negative integers $i$, if $i \leq n$ then

- if $p_{i} \notin z_{k}$ then $\alpha_{i}^{k}=0$ else $\alpha_{i}^{k}=1$.

Therefore, for all positive integers $k$, if $k \leq N$ then $\tau\left(\vec{\alpha}^{k-1}\right) \in z_{k-1}$ and $\tau\left(\vec{\alpha}^{k}\right) \in z_{k}$. Since $z_{k-1} R_{\Delta} z_{k}$, $\tau\left(\vec{\alpha}^{k-1}\right) \delta \tau\left(\vec{\alpha}^{k}\right) \in \Delta$. Consequently, $\tau\left(\vec{\alpha}^{k-1}\right) \delta \tau\left(\vec{\alpha}^{k}\right) \in \Sigma_{n}$. Thus, $\left|\left\lfloor\vec{\alpha}^{k-1}\right\rfloor-\left\lfloor\vec{\alpha}^{k}\right\rfloor\right| \leq 1$. Hence,

- $\left\lfloor\vec{\alpha}^{1}\right\rfloor \leq\left\lfloor\vec{\alpha}^{0}\right\rfloor+1$,

- ...,

- $\left\lfloor\vec{\alpha}^{N}\right\rfloor \leq\left\lfloor\vec{\alpha}^{N-1}\right\rfloor+1$.

Since $\left\lfloor\vec{\alpha}^{0}\right\rfloor=0$ and $\left\lfloor\vec{\alpha}^{N}\right\rfloor=2^{n+1}-1,2^{n+1}-1 \leq N$ : a contradiction.

Proposition 7.8. The following logic is not canonical:

- $\operatorname{val}\left(\mathcal{C}_{n 2 c}\right)$ where $\mathcal{C}_{n 2 c}$ is the class of all non-2-colourable frames.

\section{Proof:}

Let $L_{n 2 c}$ be the logic obtained by adding to $L_{\min }$ the formula $(p \cup q) \equiv t \wedge(p \cap q) \equiv f \rightarrow p \delta p \vee q \delta q$. By Proposition 6.9, $\operatorname{val}\left(\mathcal{C}_{n 2 c}\right)=L_{n 2 c}$. Let $n$ be a non-negative integer. We consider the set $\Sigma_{n}$ of formulas defined as follows:

- $\Sigma_{n}=\left\{\phi: \phi\right.$ is a formula such that $F V(\phi) \subseteq\left\{p_{0}, \ldots, p_{n}\right\}$ and $\left.\left(\mathcal{F}_{n}, V_{n}\right) \models \phi\right\}$

where $\mathcal{F}_{n}=\left(W_{n}, R_{n}\right)$ is the frame defined as follows:

- $W_{n}=\left\{a^{0}, a^{1}\right\} \cup\{2-n, \ldots, 0\} \cup\{1\} \cup\{2, \ldots, n\}$,

- $R_{n}$ is the binary relation on $W_{n}$ such that $x R_{n} y$ iff one of the following conditions is satisfied:

- $\{x, y\}=\left\{a^{0}, a^{1}\right\}$,

- $n \leq 1$ and $\{x, y\}=\left\{a^{0}, 1\right\}$,

- $n \leq 1$ and $\{x, y\}=\left\{a^{1}, 1\right\}$,

$-n \geq 2$ and $\{x, y\}=\left\{a^{0}, n\right\}$,

- $n \geq 2$ and $\{x, y\}=\left\{a^{1}, 2-n\right\}$,

$-n \geq 2, x \in\{2-n, \ldots, 0\} \cup\{1\} \cup\{2, \ldots, n\}, y \in\{2-n, \ldots, 0\} \cup\{1\} \cup\{2, \ldots, n\}$ and one of the following conditions is satisfied:

$* x \notin\{2-n, n\}, y \notin\{2-n, n\}$ and $x R_{n-1} y$,

$* x=2-n, y \notin\{2-n, n\}$ and $a^{0} R_{n-1} y$,

$* x=n, y \notin\{2-n, n\}$ and $a^{1} R_{n-1} y$, 


$$
\begin{aligned}
& * x \notin\{2-n, n\}, y=2-n \text { and } x R_{n-1} a^{0}, \\
& * x \notin\{2-n, n\}, y=n \text { and } x R_{n-1} a^{1}
\end{aligned}
$$

and $V_{n}$ is the valuation based on $\mathcal{F}_{n}$ defined as follows:

- $V_{n}$ is the function assigning to each Boolean variable $p$ the subset $V_{n}(p)$ of $W_{n}$ such that $x \in V_{n}(p)$ iff one of the following conditions is satisfied:

- $x=a^{0}$ and there exists a non-negative integer $k$ such that $k \leq n$ and $p=p_{k}$,

- $n \geq 2, x \in\{2-n, \ldots, 0\}$ and there exists a non-negative integer $k$ such that $k \leq n, p=p_{k}$ and $k \neq 2-x$,

- $x=1$ and there exists a non-negative integer $k$ such that $k \leq n, p=p_{k}$ and $k \neq 1$,

- $n \geq 2, x \in\{2, \ldots, n\}$ and there exists a non-negative integer $k$ such that $k \leq n, p=p_{k}$ and $k \geq x$.

Note that $W_{0}=\left\{a^{0}, a^{1}, 1\right\}, W_{1}=\left\{a^{0}, a^{1}, 1\right\}$ and $W_{2}=\left\{a^{0}, a^{1}, 0,1,2\right\}$.

Lemma 7.9. $\operatorname{val}\left(\mathcal{F}_{n}, L_{n 2 c}\right)$.

\section{Proof:}

It suffices to remark that $\mathcal{F}_{n}$ is non-2-colourable.

Lemma 7.10. $\left(\mathcal{F}_{n}, V_{n}\right) \models \bigcup\left\{\Sigma_{i}: i\right.$ is a non-negative integer such that $\left.i \leq n\right\}$.

\section{Proof:}

Consider a non-negative integer $i$ such that $i \leq n$. Let $\phi$ be a formula in $\Sigma_{i}$. Hence, $\phi$ is a formula such that $F V(\phi) \subseteq\left\{p_{0}, \ldots, p_{i}\right\}$ and $\left(\mathcal{F}_{i}, V_{i}\right) \models \phi$. Now, we consider the binary relation $Z$ between $W_{i}$ and $W_{n}$ defined as follows:

- $Z=\left\{\left(x_{i}, x_{n}\right): x_{i} \in W_{i}\right.$ and $x_{n} \in W_{n}$ are such that for all non-negative integers $k$, if $k \leq i$ then $x_{i} \in V_{i}\left(p_{k}\right)$ iff $\left.x_{n} \in V_{n}\left(p_{k}\right)\right\}$.

The reader may easily verify that $Z$ is a bisimulation between $\left(\mathcal{F}_{i}, V_{i}\right)$ and $\left(\mathcal{F}_{n}, V_{n}\right)$ if one restricts the language to $\left\{p_{0}, \ldots, p_{i}\right\}$. Therefore, by Proposition 3.5, $\left(\mathcal{F}_{n}, V_{n}\right) \models \phi$.

Let $\Sigma=\bigcup\left\{\Sigma_{n}: n\right.$ is a non-negative integer $\}$.

Lemma 7.11. $L_{n 2 c}+\Sigma$ is a consistent $L_{n 2 c}$-theory.

\section{Proof:}

By Lemma 7.9 and Lemma 7.10.

By Lemma 6.4, there exists a maximal consistent $L_{n 2 c}$-theory $\Delta$ such that $\Sigma \subseteq \Delta$. By Proposition 4.3, it remains to show that the canonical frame $\mathcal{F}_{\Delta}=\left(W_{\Delta}, R_{\Delta}\right)$ for $\Delta$ is 2-colourable. Let $\mathcal{F}=(W, R)$ be the frame defined as follows: 
- $W=\left\{a^{0}, a^{1}\right\} \cup\{\ldots, 0\} \cup\{1\} \cup\{2, \ldots\}$,

- $R$ is the binary relation on $W$ such that $x R y$ iff one of the following conditions is satisfied:

$-\{x, y\}=\left\{a^{0}, a^{1}\right\}$

- $x \in\{\ldots, 0\} \cup\{1\} \cup\{2, \ldots\}, y \in\{\ldots, 0\} \cup\{1\} \cup\{2, \ldots\}$ and there exists a non-negative integer $n$ such that $x R_{n} y$

and $V$ be the valuation based on $\mathcal{F}$ defined as follows:

- $V$ is the function assigning to each Boolean variable $p$ the subset $V(p)$ of $W$ such that $x \in V(p)$ iff one of the following conditions is satisfied:

- $x=a^{0}$ and there exists a non-negative integer $k$ such that $p=p_{k}$,

- $x \in\{\ldots, 0\}$ and there exists a non-negative integer $k$ such that $p=p_{k}$ and $k \neq 2-x$,

$-x=1$ and there exists a non-negative integer $k$ such that $p=p_{k}$ and $k \neq 1$,

$-x \in\{2, \ldots\}$ and there exists a non-negative integer $k$ such that $p=p_{k}$ and $k \geq x$.

Obviously, $\mathcal{F}$ is 2-colourable. Now, we consider the function $f$ from $W_{\Delta}$ to $W$ defined as follows:

- $f\left(x_{\Delta}\right)=x$ iff for all non-negative integers $k, x_{\Delta} \in V_{\Delta}\left(p_{k}\right)$ iff $x \in V\left(p_{k}\right)$.

The reader may easily verify that $f$ is an isomorphism from $\mathcal{F}_{\Delta}$ to $\mathcal{F}$. Since $\mathcal{F}$ is 2-colourable, $\mathcal{F}_{\Delta}$ is 2-colourable.

Proposition 7.12. The following logic is not canonical:

- $\operatorname{val}\left(\mathcal{C}_{\text {loo }}\right)$ where $\mathcal{C}_{\text {loo }}$ is the class of all looping frames.

\section{Proof:}

Let $L_{l o o}$ be the logic obtained by adding to $L_{\text {min }}$ the formula $(p \cap-q) \not \equiv f \rightarrow p \delta-q \vee q \delta-q$. By Proposition $6.9, \operatorname{val}\left(\mathcal{C}_{l o o}\right)=L_{l o o}$. Let $n$ be a non-negative integer. We consider the set $\Sigma_{n}$ of formulas defined as follows:

- $\Sigma_{n}=\left\{\tau(\vec{\alpha}) \delta \tau(\vec{\beta}): \vec{\alpha}\right.$ is a sequence in $\{0,1\}^{\star}$ and $\vec{\beta}$ is a sequence in $\{0,1\}^{\star}$ such that $|\vec{\alpha}|=n$, $|\vec{\beta}|=n$ and $\left.\lceil\vec{\alpha}\rceil+1=\lceil\vec{\beta}\rceil \bmod 2^{n+1}\right\} \cup\left\{\tau(\vec{\alpha}) \bar{\delta} \tau(\vec{\beta}): \vec{\alpha}\right.$ is a sequence in $\{0,1\}^{\star}$ and $\vec{\beta}$ is a sequence in $\{0,1\}^{\star}$ such that $|\vec{\alpha}|=n,|\vec{\beta}|=n$ and $\left.\lceil\vec{\alpha}\rceil+1 \neq\lceil\vec{\beta}\rceil \bmod 2^{n+1}\right\}$.

We consider the frame $\mathcal{F}_{n}=\left(W_{n}, R_{n}\right)$ defined as follows:

- $W_{n}$ is the set of all sequences $\vec{\alpha}$ in $\{0,1\}^{\star}$ such that $|\vec{\alpha}|=i$ for some non-negative integer $i$ such that $i \leq n$,

- $R_{n}$ is the binary relation on $W_{n}$ such that $\vec{\alpha} R_{n} \vec{\beta}$ iff $|\vec{\alpha}|=i,|\vec{\beta}|=i$ and $\lceil\vec{\alpha}\rceil+1=\lceil\vec{\beta}\rceil \bmod 2^{i+1}$ for some non-negative integer $i$ such that $i \leq n$

and the valuation $V_{n}$ based on $\mathcal{F}_{n}$ defined as follows: 
- $V_{n}$ is the function assigning to each Boolean variable $p$ the subset $V_{n}(p)$ of $W_{n}$ such that $\vec{\alpha} \in V_{n}(p)$ iff $|\vec{\alpha}|=i$ and there exists a non-negative integer $k$ such that $k \leq i, p=p_{k}$ and $\alpha_{k}=1$ for some non-negative integer $i$ such that $i \leq n$.

Note that $W_{0}=\{(0),(1)\}, W_{1}=\{(0),(1),(0,0),(0,1),(1,0),(1,1)\}$ and $W_{2}=\{(0),(1),(0,0),(0$, $1),(1,0),(1,1),(0,0,0),(0,0,1),(0,1,0),(0,1,1),(1,0,0),(1,0,1),(1,1,0),(1,1,1)\}$.

Lemma 7.13. $\operatorname{val}\left(\mathcal{F}_{n}, L_{l o o}\right)$.

\section{Proof:}

It suffices to remark that $\mathcal{F}_{n}$ is looping.

Lemma 7.14. $\left(\mathcal{F}_{n}, V_{n}\right) \models \bigcup\left\{\Sigma_{i}: i\right.$ is a non-negative integer such that $\left.i \leq n\right\}$.

\section{Proof:}

Consider a non-negative integer $i$ such that $i \leq n$.

Let $\tau(\vec{\alpha}) \delta \tau(\vec{\beta})$ be a formula in $\Sigma_{i}$. Hence, $\vec{\alpha}$ is a sequence in $\{0,1\}^{\star}$ and $\vec{\beta}$ is a sequence in $\{0,1\}^{\star}$ such that $|\vec{\alpha}|=i,|\vec{\beta}|=i$ and $\lceil\vec{\alpha}\rceil+1=\lceil\vec{\beta}\rceil \bmod 2^{i+1}$. Since $i \leq n,\left(\mathcal{F}_{n}, V_{n}\right) \models \tau(\vec{\alpha}) \delta \tau(\vec{\beta})$.

Let $\tau(\vec{\alpha}) \bar{\delta} \tau(\vec{\beta})$ be a formula in $\Sigma_{i}$. Hence, $\vec{\alpha}$ is a sequence in $\{0,1\}^{\star}$ and $\vec{\beta}$ is a sequence in $\{0,1\}^{\star}$ such that $|\vec{\alpha}|=i,|\vec{\beta}|=i$ and $\lceil\vec{\alpha}\rceil+1 \neq\lceil\vec{\beta}\rceil \bmod 2^{i+1}$. Since $i \leq n,\left(\mathcal{F}_{n}, V_{n}\right) \models \tau(\vec{\alpha}) \bar{\delta} \tau(\vec{\beta})$.

Let $\Sigma=\bigcup\left\{\Sigma_{n}: n\right.$ is a non-negative integer $\}$.

Lemma 7.15. $L_{l o o}+\Sigma$ is a consistent $L_{l o o}$-theory.

\section{Proof:}

By Lemma 7.13 and Lemma 7.14.

By Lemma 6.4, there exists a maximal consistent $L_{l o o}$-theory $\Delta$ such that $\Sigma \subseteq \Delta$. By Proposition 4.3, it remains to show that the canonical frame $\mathcal{F}_{\Delta}=\left(W_{\Delta}, R_{\Delta}\right)$ for $\Delta$ is not looping. Suppose $\mathcal{F}_{\Delta}$ is looping. Let $x \in W_{\Delta}$. Since $\mathcal{F}_{\Delta}$ is looping, there exists a positive integer $N$ and there exists a sequence $\left(y_{0}, \ldots, y_{N}\right)$ in $W_{\Delta}$ such that $y_{0}=x, y_{N}=x$ and for all positive integers $k$, if $k \leq N$ then $y_{k-1} R_{\Delta} y_{k}$. Let $n$ be a non-negative integer such that $N<2^{n+1}$ and $k$ be a non-negative integer such that $k \leq N$. Consider the sequence $\vec{\alpha}^{k}=\left(\alpha_{0}^{k}, \ldots, \alpha_{n}^{k}\right)$ in $\{0,1\}^{\star}$ such that for all non-negative integers $i$, if $i \leq n$ then

- if $p_{i} \notin y_{k}$ then $\alpha_{i}^{k}=0$ else $\alpha_{i}^{k}=1$.

Therefore, for all positive integers $k$, if $k \leq N$ then $\tau\left(\vec{\alpha}^{k-1}\right) \in y_{k-1}$ and $\tau\left(\vec{\alpha}^{k}\right) \in y_{k}$. Since $y_{k-1} R_{\Delta} y_{k}$, $\tau\left(\vec{\alpha}^{k-1}\right) \delta \tau\left(\vec{\alpha}^{k}\right) \in \Delta$. Consequently, $\tau\left(\vec{\alpha}^{k-1}\right) \delta \tau\left(\vec{\alpha}^{k}\right) \in \Sigma_{n}$. Thus, $\left\lceil\vec{\alpha}^{k-1}\right\rceil+1=\left\lceil\vec{\alpha}^{k}\right\rceil \bmod 2^{n+1}$. Hence,

- $\left\lceil\vec{\alpha}^{0}\right\rceil+1=\left\lceil\vec{\alpha}^{1}\right\rceil \bmod 2^{n+1}$,

- ...,

- $\left\lceil\vec{\alpha}^{N-1}\right\rceil+1=\left\lceil\vec{\alpha}^{N}\right\rceil \bmod 2^{n+1}$.

Since $\left\lceil\vec{\alpha}^{0}\right\rceil=\left\lceil\vec{\alpha}^{N}\right\rceil, N=0 \bmod 2^{n+1}$. Since $N<2^{n+1}, N=0$ : a contradiction. 


\section{Compatible formulas}

Now, we introduce the concept of compatible formula.

\section{Definition 8.1. (Compatible formulas)}

Let $L$ be a logic and $\phi$ be a formula. We shall say that $\phi$ is compatible with $L$ iff there exists a positive first-order formula $\alpha\left(u_{1}, \ldots, u_{k}\right)$ in $\mathcal{L}^{1}(\emptyset)$ and there exists a quantifier-free positive first-order formula $\beta\left(u_{1}, \ldots, u_{k}, v_{1}, \ldots, v_{l}\right)$ in $\mathcal{L}^{1}(\emptyset)$ such that for all frames $\mathcal{F}$, if $\operatorname{val}(\mathcal{F}, L)$ then $\operatorname{val}(\mathcal{F}, \phi)$ iff $\mathcal{F} \models$ $\forall u_{1} \ldots \forall u_{k}\left(\alpha\left(u_{1}, \ldots, u_{k}\right) \rightarrow \exists v_{1} \ldots \exists v_{l} \beta\left(u_{1}, \ldots, u_{k}, v_{1}, \ldots, v_{l}\right)\right)$.

Take the case of the formulas $p \not \equiv f \rightarrow p \delta p$ and $p \delta q \rightarrow p \delta r \vee-r \delta q$. They are compatible with $L_{\text {min }}$ because according to Proposition 4.3, they correspond to the first-order sentences $\forall u R_{\delta}(u, u)$ and $\forall u \forall v\left(R_{\delta}(u, v) \rightarrow \exists w\left(R_{\delta}(u, w) \wedge R_{\delta}(w, v)\right)\right)$ within the class of all frames. Nevertheless, we still do not know if the following decision problem is decidable:

input: a formula $\phi$,

output: determine whether $\phi$ is compatible with $L_{\min }$.

Our conjecture is that the above decision problem is undecidable. Now, let us prove the

Proposition 8.2. Let $L$ be a logic and $\Phi$ be a set of formulas such that for all $\phi \in \Phi, \phi$ is compatible with $L$. If $L$ is canonical then $L_{\Phi}$ is canonical.

Proof:

Suppose $L$ is canonical and $L_{\Phi}$ is not canonical. Since $L_{\Phi}$ is not canonical, there exists a maximal consistent $L_{\Phi}$-theory $\Gamma$ such that not $\operatorname{val}\left(\mathcal{F}_{\Gamma}, L_{\Phi}\right) . \quad$ Hence, not $\operatorname{val}\left(\mathcal{F}_{\Gamma}, L\right)$ or not $\operatorname{val}\left(\mathcal{F}_{\Gamma}, \Phi\right)$. Since $\Gamma$ is a maximal consistent $L_{\Phi}$-theory, $\Gamma$ is a maximal consistent $L$-theory. Since $L$ is canonical, $\operatorname{val}\left(\mathcal{F}_{\Gamma}, L\right)$. Since not $\operatorname{val}\left(\mathcal{F}_{\Gamma}, L\right)$ or not $\operatorname{val}\left(\mathcal{F}_{\Gamma}, \Phi\right)$, not $\operatorname{val}\left(\mathcal{F}_{\Gamma}, \Phi\right)$. Thus, there exists $\phi \in \Phi$ such that not $\operatorname{val}\left(\mathcal{F}_{\Gamma}, \phi\right)$. Since $\phi \in \Phi, \phi$ is compatible with $L$. Therefore, there exists a positive first-order formula $\alpha\left(u_{1}, \ldots, u_{k}\right)$ in $\mathcal{L}^{1}(\emptyset)$ and there exists a quantifier-free positive first-order formula $\beta\left(u_{1}, \ldots, u_{k}, v_{1}, \ldots, v_{l}\right)$ in $\mathcal{L}^{1}(\emptyset)$ such that for all frames $\mathcal{F}$, if $\operatorname{val}(\mathcal{F}, L)$ then $\operatorname{val}(\mathcal{F}, \phi)$ iff $\mathcal{F} \models \forall u_{1} \ldots \forall u_{k}\left(\alpha\left(u_{1}, \ldots, u_{k}\right) \rightarrow \exists v_{1} \ldots \exists v_{l} \beta\left(u_{1}, \ldots, u_{k}, v_{1}, \ldots, v_{l}\right)\right)$. Since $\operatorname{val}\left(\mathcal{F}_{\Gamma}, L\right)$ and not $\operatorname{val}\left(\mathcal{F}_{\Gamma}, \phi\right), \mathcal{F}_{\Gamma} \not \forall \forall u_{1} \ldots \forall u_{k}\left(\alpha\left(u_{1}, \ldots, u_{k}\right) \rightarrow \exists v_{1} \ldots \exists v_{l} \beta\left(u_{1}, \ldots, u_{k}, v_{1}, \ldots, v_{l}\right)\right)$. Consequently, there exists $x_{1}, \ldots, x_{k}$ in $W_{\Gamma}$ such that $\mathcal{F}_{\Gamma} \not \forall \alpha\left(u_{1}, \ldots, u_{k}\right) \rightarrow \exists v_{1} \ldots \exists v_{l} \beta\left(u_{1}, \ldots, u_{k}, v_{1}, \ldots, v_{l}\right)$ $\left[x_{1}, \ldots, x_{k}\right]$. Hence, $\mathcal{F}_{\Gamma} \models \alpha\left(u_{1}, \ldots, u_{k}\right)\left[x_{1}, \ldots, x_{k}\right]$ and $\mathcal{F}_{\Gamma} \not \forall \exists v_{1} \ldots \exists v_{l} \beta\left(u_{1}, \ldots, u_{k}, v_{1}, \ldots, v_{l}\right)$ $\left[x_{1}, \ldots, x_{k}\right]$. Now, take a non-negative integer $n$. Let $\mathcal{M}_{\Gamma}^{n}=\left(W_{\Gamma}^{n}, R_{\Gamma}^{n}, V_{\Gamma}^{n}\right)$ be the filtration of $\mathcal{M}_{\Gamma}=\left(W_{\Gamma}, R_{\Gamma}, V_{\Gamma}\right)$ through $B V_{n}=\left\{p_{0}, \ldots, p_{n-1}\right\}$ and $\mathcal{F}_{\Gamma}^{n}=\left(W_{\Gamma}^{n}, R_{\Gamma}^{n}\right)$ be the corresponding frame. By Proposition 5.8, $\mathcal{F}_{\Gamma}^{n}$ is a homomorphic image of $\mathcal{F}_{\Gamma}$. To see this, it suffices to take $B V^{\prime}=B V_{n}$ and $B V^{\prime \prime}=B V$ for the sets of Boolean variables considered in Proposition 5.8. Since $\alpha\left(u_{1}, \ldots, u_{k}\right)$ is a positive first-order formula such that $\mathcal{F}_{\Gamma} \models \alpha\left(u_{1}, \ldots, u_{k}\right)\left[x_{1}, \ldots, x_{k}\right], \mathcal{F}_{\Gamma}^{n} \models \alpha\left(u_{1}, \ldots, u_{k}\right)\left[\left|x_{1}\right|_{B V_{n}}\right.$, $\left.\ldots,\left|x_{k}\right|_{B V_{n}}\right]$. To see this, it suffices to reason by induction on $\alpha\left(u_{1}, \ldots, u_{k}\right)$. Since $\Gamma$ is a maximal consistent $L_{\Phi}$-theory, by Proposition 6.7, $\operatorname{val}\left(\mathcal{F}_{\Gamma}^{n}, L_{\Phi}\right)$. Thus, $\operatorname{val}\left(\mathcal{F}_{\Gamma}^{n}, L\right)$ and $\operatorname{val}\left(\mathcal{F}_{\Gamma}^{n}, \Phi\right)$. Since $\operatorname{val}\left(\mathcal{F}_{\Gamma}^{n}, L\right)$ and for all frames $\mathcal{F}$, if $\operatorname{val}(\mathcal{F}, L)$ then $\operatorname{val}(\mathcal{F}, \phi)$ iff $\mathcal{F} \models \forall u_{1} \ldots \forall u_{k}\left(\alpha\left(u_{1}, \ldots, u_{k}\right) \rightarrow\right.$ $\left.\exists v_{1} \ldots \exists v_{l} \beta\left(u_{1}, \ldots, u_{k}, v_{1}, \ldots, v_{l}\right)\right), \operatorname{val}\left(\mathcal{F}_{\Gamma}^{n}, \phi\right)$ iff $\mathcal{F}_{\Gamma}^{n} \models \forall u_{1} \ldots \forall u_{k}\left(\alpha\left(u_{1}, \ldots, u_{k}\right) \rightarrow \exists v_{1} \ldots\right.$ $\left.\exists v_{l} \beta\left(u_{1}, \ldots, u_{k}, v_{1}, \ldots, v_{l}\right)\right)$. Since $\operatorname{val}\left(\mathcal{F}_{\Gamma}^{n}, \Phi\right)$ and $\phi \in \Phi, \operatorname{val}\left(\mathcal{F}_{\Gamma}^{n}, \phi\right)$. Since $\operatorname{val}\left(\mathcal{F}_{\Gamma}^{n}, \phi\right)$ iff $\mathcal{F}_{\Gamma}^{n} \models$ 
$\forall u_{1} \ldots \forall u_{k}\left(\alpha\left(u_{1}, \ldots, u_{k}\right) \rightarrow \exists v_{1} \ldots \exists v_{l} \beta\left(u_{1}, \ldots, u_{k}, v_{1}, \ldots, v_{l}\right)\right), \mathcal{F}_{\Gamma}^{n} \models \forall u_{1} \ldots \forall u_{k}\left(\alpha\left(u_{1}, \ldots, u_{k}\right)\right.$ $\left.\rightarrow \exists v_{1} \ldots \exists v_{l} \beta\left(u_{1}, \ldots, u_{k}, v_{1}, \ldots, v_{l}\right)\right)$. Therefore, $\mathcal{F}_{\Gamma}^{n} \models \alpha\left(u_{1}, \ldots, u_{k}\right) \rightarrow \exists v_{1} \ldots \exists v_{l} \beta\left(u_{1}, \ldots, u_{k}\right.$, $\left.v_{1}, \ldots, v_{l}\right)\left[\left|x_{1}\right|_{B V_{n}}, \ldots,\left|x_{k}\right|_{B V_{n}}\right]$. Since $\mathcal{F}_{\Gamma}^{n} \models \alpha\left(u_{1}, \ldots, u_{k}\right)\left[\left|x_{1}\right|_{B V_{n}}, \ldots,\left|x_{k}\right|_{B V_{n}}\right], \mathcal{F}_{\Gamma}^{n} \models$ $\exists v_{1} \ldots \exists v_{l} \beta\left(u_{1}, \ldots, u_{k}, v_{1}, \ldots, v_{l}\right)\left[\left|x_{1}\right|_{B V_{n}}, \ldots,\left|x_{k}\right|_{B V_{n}}\right]$. Consequently, there exists $y_{1}^{n}, \ldots, y_{l}^{n}$ in $W_{\Gamma}$ such that $\mathcal{F}_{\Gamma}^{n} \models \beta\left(u_{1}, \ldots, u_{k}, v_{1}, \ldots, v_{l}\right)\left[\left|x_{1}\right|_{B V_{n}}, \ldots,\left|x_{k}\right|_{B V_{n}},\left|y_{1}^{n}\right|_{B V_{n}}, \ldots,\left|y_{l}^{n}\right|_{B V_{n}}\right]$.

To summarize, we have shown that for all non-negative integers $n, \mathcal{F}_{\Gamma}^{n} \models \beta\left(u_{1}, \ldots, u_{k}, v_{1}, \ldots, v_{l}\right)[\mid$ $\left.\left.x_{1}\right|_{B V_{n}}, \ldots,\left|x_{k}\right|_{B V_{n}},\left|y_{1}^{n}\right|_{B V_{n}}, \ldots,\left|y_{l}^{n}\right|_{B V_{n}}\right]$. By Proposition 5.8, $\mathcal{F}_{\Gamma}^{n}$ is a homomorphic image of $\mathcal{F}_{\Gamma}^{n+1}$. To see this, it suffices to take $B V^{\prime}=B V_{n}$ and $B V^{\prime \prime}=B V_{n+1}$ for the sets of Boolean variables considered in Proposition 5.8. Since $\beta\left(u_{1}, \ldots, u_{k}, v_{1}, \ldots, v_{l}\right)$ is a positive first-order formula such that for all non-negative integers $n, \mathcal{F}_{\Gamma}^{n} \models \beta\left(u_{1}, \ldots, u_{k}, v_{1}, \ldots, v_{l}\right)\left[\left|x_{1}\right|_{B V_{n}}, \ldots,\left|x_{k}\right|_{B V_{n}}, \mid\right.$ $\left.\left.y_{1}^{n}\right|_{B V_{n}}, \ldots,\left|y_{l}^{n}\right|_{B V_{n}}\right],(\star)$ for all non-negative integers $n, \mathcal{F}_{\Gamma}^{n} \models \beta\left(u_{1}, \ldots, u_{k}, v_{1}, \ldots, v_{l}\right)\left[\left|x_{1}\right|_{B V_{n}}\right.$, $\left.\ldots,\left|x_{k}\right|_{B V_{n}},\left|y_{1}^{n+1}\right|_{B V_{n}}, \ldots,\left|y_{l}^{n+1}\right|_{B V_{n}}\right]$. To see this, it suffices to reason by induction on $\beta\left(u_{1}, \ldots, u_{k}, v_{1}, \ldots, v_{l}\right)$. Let $T_{l}$ be the tree defined as follows:

(i) the root of $T_{l}$ is labelled by the $l$-tuple $(\epsilon, \ldots, \epsilon)$ where $\epsilon$ denotes the 0 -tuple of bits and

(ii) the successors of a node of $T_{l}$ at depth $n$ labelled by the $l$-tuple $\left(\left(b_{1,0}, \ldots, b_{1, n-1}\right), \ldots,\left(b_{l, 0}, \ldots\right.\right.$, $\left.\left.b_{l, n-1}\right)\right)$ of $n$-tuples of bits are the $2^{l}$ nodes of $T_{l}$ at depth $n+1$ labelled by the $l$-tuple $\left(\left(b_{1,0}, \ldots\right.\right.$, $\left.\left.b_{1, n-1}, b_{1, n}\right), \ldots,\left(b_{l, 0}, \ldots, b_{l, n-1}, b_{l, n}\right)\right)$ of $(n+1)$-tuples of bits. By definition, remark that $T_{l}$ is infinite and finitely branching. Let $T_{l}^{\prime}$ be the least rooted subtree of $T_{l}$ containing for all non-negative integers $n$, the nodes of $T_{l}$ at depth $n$ corresponding to all possible $l$-tuples $y_{1}^{n}, \ldots, y_{l}^{n}$. By $(\star)$, remark that $T_{l}^{\prime}$ is infinite and finitely branching. By König's Infinity Lemma for Trees [30, Chapter 1], $T_{l}^{\prime}$ has an infinite branch. Let $y_{1}, \ldots, y_{l}$ be the elements in $W_{\Gamma}$ corresponding to this infinite branch. Let $n$ be a non-negative integer such that the restriction $\mathcal{F}_{\Gamma}^{n, \star}=\left(W_{\Gamma}^{n, \star}, R_{\Gamma}^{n, \star}\right)$ of $\mathcal{F}_{\Gamma}^{n}$ to $\left\{\left|x_{1}\right|_{B V_{n}}\right.$, $\left.\ldots,\left|x_{k}\right|_{B V_{n}},\left|y_{1}\right|_{B V_{n}}, \ldots,\left|y_{l}\right|_{B V_{n}}\right\}$ is isomorphic to the restriction $\mathcal{F}_{\Gamma}^{\star}=\left(W_{\Gamma}^{\star}, R_{\Gamma}^{\star}\right)$ of $\mathcal{F}_{\Gamma}$ to $\left\{x_{1}, \ldots, x_{k}, y_{1}, \ldots, y_{l}\right\}$. By construction, $\left|y_{1}\right|_{B V_{n}}=\left|y_{1}^{n}\right|_{B V_{n}}, \ldots,\left|y_{l}\right|_{B V_{n}}=\left|y_{l}^{n}\right|_{B V_{n}}$. Since $\mathcal{F}_{\Gamma}^{n} \models \beta\left(u_{1}, \ldots, u_{k}, v_{1}, \ldots, v_{l}\right)\left[\left|x_{1}\right|_{B V_{n}}, \ldots,\left|x_{k}\right|_{B V_{n}},\left|y_{1}^{n}\right|_{B V_{n}}, \ldots,\left|y_{l}^{n}\right|_{B V_{n}}\right], \mathcal{F}_{\Gamma}^{n} \models \beta\left(u_{1}, \ldots\right.$, $\left.u_{k}, v_{1}, \ldots, v_{l}\right)\left[\left|x_{1}\right|_{B V_{n}}, \ldots,\left|x_{k}\right|_{B V_{n}},\left|y_{1}\right|_{B V_{n}}, \ldots,\left|y_{l}\right|_{B V_{n}}\right]$. Since $\beta\left(u_{1}, \ldots, u_{k}, v_{1}, \ldots, v_{l}\right)$ is a quantifier-free first-order formula, $\mathcal{F}_{\Gamma}^{n, \star} \models \beta\left(u_{1}, \ldots, u_{k}, v_{1}, \ldots, v_{l}\right)\left[\left|x_{1}\right|_{B V_{n}}, \ldots,\left|x_{k}\right|_{B V_{n}},\left|y_{1}\right|_{B V_{n}}\right.$, $\left.\ldots,\left|y_{l}\right|_{B V_{n}}\right]$. To see this, it suffices to reason by induction on $\beta\left(u_{1}, \ldots, u_{k}, v_{1}, \ldots, v_{l}\right)$. Since $\mathcal{F}_{\Gamma}^{n, \star}$ is isomorphic to $\mathcal{F}_{\Gamma}^{\star}, \mathcal{F}_{\Gamma}^{\star}=\beta\left(u_{1}, \ldots, u_{k}, v_{1}, \ldots, v_{l}\right)\left[x_{1}, \ldots, x_{k}, y_{1}, \ldots, y_{l}\right]$. Since $\beta\left(u_{1}, \ldots, u_{k}, v_{1}, \ldots, v_{l}\right)$ is a quantifier-free first-order formula, $\mathcal{F}_{\Gamma} \models \beta\left(u_{1}, \ldots, u_{k}, v_{1}, \ldots, v_{l}\right)\left[x_{1}, \ldots, x_{k}, y_{1}, \ldots, y_{l}\right]$. To see this, it suffices to reason by induction on $\beta\left(u_{1}, \ldots, u_{k}, v_{1}, \ldots, v_{l}\right)$. Hence, $\mathcal{F}_{\Gamma} \models \exists v_{1} \ldots \exists v_{l} \beta\left(u_{1}\right.$, $\left.\ldots, u_{k}, v_{1}, \ldots, v_{l}\right)\left[x_{1}, \ldots, x_{k}\right]:$ a contradiction.

Remark that Proposition 7.3 is an immediate consequence of the more general result stated in Proposition 8.2. An immediate corollary of Proposition 8.2 is the

Corollary 8.3. Let $L$ be a logic and $\phi$ be a formula such that $\phi$ is compatible with $L$. If $L$ is canonical then $L_{\{\phi\}}$ is canonical.

By contrast, we do not know if there exists logics $L$ and formulas $\phi$ such that $\phi$ is not compatible with $L, L$ is canonical and $L_{\{\phi\}}$ is canonical. 


\section{Conclusion}

In Section 4, we provided classes of formulas defining first-order or second-order conditions on frames. For pointers to this line of work in the basic modal language, see Goldblatt and Thomason [19]. A Goldblatt-Thomason theorem for our language is still to be obtained. In Section 7, we mentioned our conjecture that it is undecidable whether a given formula $\phi$ is compatible with $L_{\min }$. For pointers to this line of work in the basic modal language, see Chagrova's theorem in [8]. A Chagrova's theorem for our language is still to be obtained. In modal logic, Sahlqvist formulas are modal formulas with remarkable properties [24, 25]: the Sahlqvist correspondence theorem says that every Sahlqvist formula corresponds to a first-order definable class of frames; the Sahlqvist completeness theorem says that when Sahlqvist formulas are used as axioms in a normal logic, the logic is complete with respect to the elementary class of frames the axioms define. Then, in the end, a natural question is to ask whether a Sahlqvist-like theory can be elaborated for our language. A first answer to this question has been presented in [1].

\section{Acknowledgements}

We make a point of thanking Dimiter Vakarelov for many stimulating discussions in the field of Boolean logic with relations. We also wish to thank the anonymous referees for their useful comments and their detailed suggestions about the overall organisation of the paper and for pointing out typos we had missed. Our work has been supported by the University of Sofia (contract 63/2009) and the Bulgarian National Science Fund (contract DID02/32/2009).

\section{References}

[1] Balbiani, P., Kikot, S.: Sahlqvist theorems for precontact logics. In: Advances in Modal Logic. Volume 9. College Publications (2012) 55-70.

[2] Balbiani, P., Tinchev, T.: Definability over the class of all partitions. Journal of Logic and Computation 16 (2006) 541-557

[3] Balbiani, P., Tinchev, T.: Boolean logics with relations. Journal of Logic and Algebraic Programming 79 (2010) 707-721.

[4] Balbiani, P., Tinchev, T., Vakarelov, D.: Dynamic logics of the region-based theory of discrete spaces. Journal of Applied Non-Classical Logics 17 (2007) 39-61.

[5] Balbiani, P., Tinchev, T., Vakarelov, D.: Modal logics for region-based theories of space. Fundamenta Informaticæ81 (2007) 29-82.

[6] Biacino, L., Gerla, G.: Connection structures: Grzegorczyk's and Whitehead's definition of point. Notre Dame Journal of Formal Logic 37 (1996) 431-439.

[7] Blackburn, P., de Rijke, M., Venema, Y.: Modal Logic. Cambridge University Press (2001).

[8] Chagrov, A., Chagrova, L.: The truth about algorithmic problems in correspondence theory. In: Advances in Modal Logic. Volume 6. College Publications (2006) 121-138.

[9] Chagrov, A., Zakharyaschev, M.: Modal Logic. Oxford University Press (1997).

[10] Clarke, B.: A calculus of individuals based on “connection”. Notre Dame Journal of Formal Logic 22 (1981) 204-219. 
[11] Cohn, A., Hazarika, S.: Qualitative spatial representation and reasoning: an overview. Fundamenta Informaticæ46 (2001) 1-29.

[12] Dimov, G., Vakarelov, D.: Contact algebras and region-based theory of space: a proximity approach - I. Fundamenta Informaticæ74 (2006) 209-249.

[13] Dimov, G., Vakarelov, D.: Contact algebras and region-based theory of space: proximity approach - II. Fundamenta Informaticæ74 (2006) 251-282.

[14] Düntsch, I., Vakarelov, D.: Region-based theory of discrete spaces: a proximity approach. Annals of Mathematics and Artificial Intelligence 49 (2007) 5-14.

[15] Düntsch, I., Wang, H., McCloskey, S..: A relational algebraic approach to Region Connection Calculus. Theoretical Computer Science 255 (2001) 63-83.

[16] Düntsch, I., Winter, M.: A representation theorem for Boolean contact algebras. Theoretical Computer Science 347 (2005) 498-512.

[17] Galton, A.: The mereotopology of discrete space. In: Spatial Information Theory. Springer (1999) 251-266.

[18] Galton, A.: Qualitative Spatial Change. Oxford University Press (2000).

[19] Goldblatt, R., Thomason, S.: Axiomatic classes in propositional modal logic. In: Algebra and Logic. Springer (1975) 163-173.

[20] Grädel, E., Kolaitis, P., Vardi, M.: On the decision problem for two-variable first-order logic. Bulletin of Symbolic Logic 3 (1997) 53-69.

[21] Grzegorczyk, A.: Axiomatization of geometry without points. Synthese 12 (1960) 228-235.

[22] De Laguna, T.: Point, line and surface, as sets of solids. The Journal of Philosophy 19 (1922) 449-461.

[23] Mortimer, M.: On languages with two variables. Zeitschrift für mathematische Logik und Grundlagen der Mathematik 21 (1975) 135-140.

[24] Sahlqvist, H.: Completeness and correspondence in the first and second order semantics for modal logic. In: Proceedings of the Third Scandinavian Logic Symposium. North-Holland (1975) 110-143.

[25] Sambin, G., Vaccaro, V.: A new proof of Sahlqvist theorem on modal definability and completeness. The Journal of Symbolic Logic 54 (1989) 992-999.

[26] Stell, J.: Boolean connection algebras: a new approach to the region-connection calculus. Artificial Intelligence 122 (2000) 111-136.

[27] Vakarelov, D.: Region-based theory of space: algebras of regions, representation theory, and logics. In: Mathematical Problems from Applied Logic. Logics for the XXIst Century. II. Springer (2007) 267-348.

[28] Vakarelov, D., Dimov, G., Düntsch, I., Bennett, B.: A proximity approach to some region-based theory of space. Journal of Applied Non-Classical Logics 12 (2002) 527-559.

[29] De Vries, H.: Compact Spaces and Compactifications: an Algebraic Approach. Van Gorcum (1962).

[30] Wechler, W.: Universal Algebra for Computer Scientists. Springer (1992).

[31] Whitehead, A.: Process and Reality. MacMillan (1929). 